TO APPEAR IN THE ASTROPHYSICAL JOURNAL 2010 MAY 10 V714 ISSUE

Preprint typeset using $\mathrm{LT}_{\mathrm{E}} \mathrm{X}$ style emulateapj v. 4/12/04

\title{
AN EMPIRICAL CHARACTERIZATION OF EXTENDED COOL GAS AROUND GALAXIES USING MG II ABSORPTION FEATURES ${ }^{1}$
}

\author{
Hsiao-Wen Chen ${ }^{2}$, Jennifer E. Helsb ${ }^{2}$, Jean-René Gauthier $^{2,3}$, Stephen A. Shectman $^{3}$, Ian B. Thompson ${ }^{3}, \&$ Jeremy L. $^{4}$. \\ TINKER $^{4}$ \\ To appear in the Astrophysical Journal 2010 May 10 v714 issue
}

\begin{abstract}
We report results from a survey of $\mathrm{Mg}$ II absorbers in the spectra of background QSOs that are within close angular distances to a foreground galaxy at $z<0.5$, using the Magellan Echellette Spectrograph. We have established a spectroscopic sample of 94 galaxies at a median redshift of $\langle z\rangle=0.24$ in fields around 70 distant background QSOs $\left(z_{\mathrm{QSO}}>0.6\right), 71$ of which are in an 'isolated' environment with no known companions and located at $\rho \lesssim 120 \mathrm{~h}^{-1} \mathrm{kpc}$ from the line of sight of a background QSO. The rest-frame absolute $B$-band magnitudes span a range from $M_{B}-5 \log h=-16.4$ to $M_{B}-5 \log h=-21.4$ and rest-frame $B_{A B}-R_{A B}$ colors range from $B_{A B}-R_{A B} \approx 0$ to $B_{A B}-R_{A B} \approx 1.5$. Of these 'isolated' galaxies, we find that 47 have corresponding Mg II absorbers in the spectra of background QSOs and rest-frame absorption equivalent width $W_{r}(2796)=0.1-2.34$ $\AA$, and 24 do not give rise to $\mathrm{Mg}$ II absorption to sensitive upper limits. Our analysis shows that (1) $W_{r}(2796)$ declines with increasing distance from 'isolated' galaxies but shows no clear trend in 'group' environments; (2) more luminous galaxies possess more extended $\mathrm{Mg}$ II absorbing halos with the gaseous radius scaled by $B$-band luminosity according to $R_{\text {gas }}=75 \times\left(L_{B} / L_{B_{*}}\right)^{(0.35 \pm 0.03)} h^{-1} \mathrm{kpc}$; (3) there is little dependence between the observed absorber strength and galaxy intrinsic colors; and (4) within $R_{\text {gas }}$, we find a mean covering fraction of $\left\langle\kappa_{0.3}\right\rangle \approx 70 \%$ for absorbers of $W_{r}(2796) \geq 0.3 \AA$ and $\left\langle\kappa_{0.1}\right\rangle \approx 80 \%$ for absorbers of $W_{r}(2796) \geq 0.1 \AA$. The results confirm that extended $\mathrm{Mg}$ II absorbing halos are a common and generic feature around ordinary galaxies and that the gaseous radius is a fixed fraction of the dark matter halo radius. The lack of correlation between $W_{r}(2796)$ strength and galaxy colors suggests a lack of physical connection between the origin of extended $\mathrm{Mg}$ II halos and recent star formation history of the galaxies. Finally, we discuss the total gas mass in galactic halos as traced by Mg II absorbers. We also compare our results with previous studies.
\end{abstract}

Subject headings: cosmology: observations_-intergalactic medium—quasars: absorption lines

\section{INTRODUCTION}

The 'forest' of absorption-line systems observed in the spectra of background quasars offers a sensitive probe of otherwise invisible gaseous structures in the universe, where the majority of baryons reside. Depending on the physical conditions of the absorbing gas, different transitions are expected to display different strengths and kinematic signatures. Combining QSO absorption-line observations and faint galaxy surveys, in principle, provides a unique and powerful means of establishing a comprehensive picture for understanding the growth of galaxies.

The Mg II $\lambda \lambda 2796,2803$ doublets are among the absorption features commonly seen in the spectra of distant quasars. These absorbers are understood to originate in photo-ionized gas of temperature $T \sim 10^{4} \mathrm{~K}$ (Bergeron \& Stasínska 1986; Charlton et al. 2003) and trace high-column density clouds of neutral hydrogen column density $N(\mathrm{HI}) \approx 10^{18}-10^{22} \mathrm{~cm}^{-2}$ (Rao et al. 2006). At redshifts $z=0.4-2.5$, these doublet features are redshifted into the optical spectral range and are routinely detected in the spectra of background QSOs using optical spectrographs on the ground. Over the past two decades, a large number of studies have been carried out to

\footnotetext{
${ }^{1}$ This paper includes data gathered with the 6.5 meter Magellan Telescopes located at Las Campanas Observatory, Chile.

2 Dept. of Astronomy \& Astrophysics and Kavli Institute for Cosmological Physics, University of Chicago, Chicago, IL, 60637, U.S.A. hchen@oddjob.uchicago.edu

${ }^{3}$ The Observatories of Carnegie Institution for Science, 813 Santa Barbara St., Pasadena, CA 91101

4 Berkeley Center for Cosmological Physics, University of CaliforniaBerkeley
}

characterize the statistical properties of $\mathrm{Mg}$ II absorbers, including the frequency distribution function, redshift evolution of the absorber number density, and kinematic signatures (e.g. Lanzetta et al. 1987; Sargent et al. 1988; Petitjean \& Bergeron 1990; Steidel \& Sargent 1992; Charlton \& Churchill 1998; Churchill et al. 2000, 2003; Nestor et al. 2005; Prochter et al. 2006). While the accuracy and precision of these various measurements increase with increasing sample size, the utility of known absorber statistics in advancing our understanding of galaxy evolution has been limited due to an ambiguous origin of these absorbers.

A necessary first step toward the goal of applying QSO absorption-line systems for probing the growth of baryonic structures is to understand and quantify their correlation with galaxies. The large associated HI column density (Rao et al. 2006) suggests that Mg II absorbers are similar to those H I clouds seen around individual galaxies in $21 \mathrm{~cm}$ surveys (e.g. Doyle et al. 2005). A direct connection of these absorbers to galaxies is also supported by the presence of luminous galaxies at projected distances $\rho=50-100 h^{-1} \mathrm{kpc}$ from known Mg II absorbers (Bergeron 1986; Lanzetta \& Bowen 1990, 1992; Steidel et al. 1994; Zibetti et al. 2005; Nestor et al. 2007; Kacprzak et al. 2007). However, uncertainties remain, because some galaxies found at $\rho<50 h^{-1} \mathrm{kpc}$ from a QSO sightline do not produce a corresponding Mg II absorber (e.g. Tripp \& Bowen 2005; Churchill et al. 2007), implying that $\mathrm{Mg}$ II absorbers may not probe a representative population of galaxies or simply that the gas covering fraction is not unity.

Over the past two years, we have been conducting a program that combines empirical observations and a phenomeno- 
logical model study to establish a comprehensive description of the correlation between galaxies and cool gas $\left(T \sim 10^{4}\right.$ $\mathrm{K})$ probed by $\mathrm{Mg}$ II absorbers. In Tinker \& Chen (2008), we have introduced a novel technique that adopts the halo occupation framework (e.g. Seljak 2000; Scoccimarro et al. 2001; Berlind \& Weinberg 2002) and characterizes the origin of QSO absorption-line systems based on a conditional mass function of dark matter halos in which the absorbers are found. This technique is purely statistical in nature. It characterizes the cold and warm-hot gas in dark matter halos by comparing the frequency distribution function and clustering amplitude of QSO absorbers with those of dark matter halos.

Our initial halo occupation model is constrained by known statistical properties of $\mathrm{Mg}$ II absorbers at $\langle z\rangle=0.6$, including an isothermal density profile for describing the spatial distribution of cold gas in individual dark matter halos, the frequency distribution function (e.g. Steidel \& Sargent 1992; Nestor et al. 2005; Prochter et al. 2006), and the clustering amplitude (Bouché et al. 2006; Gauthier et al. 2009; Lundgren et al. 2009). The adopted isothermal model is supported by empirical data (Chen \& Tinker 2008). The product of the halo occupation analysis is an occupation function (or "mass function") that characterizes the fractional contribution of dark matter halos (galaxies) of different masses to the observed Mg II absorbers of different strength.

Our analysis has shown that in order to reproduce the observed overall strong clustering of the absorbers, roughly $5-10 \%$ of the gas in halos up to $10^{14} h^{-1} \mathrm{M}_{\odot}$ is required to be cold. The inferred presence of cool gas in massive halos is also supported by (i) the observed strong crosscorrelation amplitude of $\mathrm{Mg}$ II absorbers and luminous red galaxies (LRG) on projected distance scales of $r_{p}<300 \mathrm{~h}^{-1}$ comoving kpc (Gauthier et al. 2009) and by (ii) direct detections of Mg II absorbers at $\lesssim 300 h^{-1} \mathrm{kpc}$ and $\lesssim 320 \mathrm{~km} \mathrm{~s}^{-1}$ from five LRGs (Gauthier et al. 2010). These LRGs are understood to reside in $>10^{13} h^{-1} \mathrm{M}_{\odot}$ halos (e.g. Blake et al. 2008; Gauthier et al. 2009). For lower-mass halos, our halo occupation analysis has shown that the incidence and covering fraction of extended cool gas is high. Therefore these halos contribute significantly to the observed Mg II statistics. The large gas covering fraction is consistent with the empirical findings of Steidel et al. (1994) and Chen \& Tinker (2008), though other authors have reported a lower covering fraction from different surveys (e.g. Tripp \& Bowen 2005; Kacprzak et al. 2008; Barton \& Cooke 2009). In summary, the initial results of our halo occupation analysis demonstrate that combining galaxy and absorber survey data together with a simple semi-analytic model already produces unique empirical constraints for contemporary theoretical models that study the gas content of dark matter halos (e.g. Mo \& Miralda-Escudé 1996; Birnboim \& Dekel 2003; Maller \& Bullock 2004; Kereš et al. 2005; 2009; Dekel \& Birnboim 2006; Birnboim et al. 2007).

In Tinker \& Chen (2010), we have expanded upon our initial halo occupation analysis to incorporate the observed number density evolution of the absorbers (Nestor et al. 2005; Prochter et al. 2006) as an additional constraint to gain insight into the redshift evolution of extended gas around galaxies. In order to incorporate the expected redshift evolution of the dark matter halo population to explain the observed number density evolution of $\mathrm{Mg}$ II absorbers, we have found that the gaseous halos must evolve with respect to their host dark matter halos. An explicit prediction of our halo occupa- tion model is a more pronounced inverse-correlation between the mean halo mass and absorber strength (e.g. Bouché et al. 2006; Gauthier et al. 2009; Lundgren et al. 2009) at $z \lesssim 0.3$ and a positive correlation between the mean halo mass and absorber strength at $z \gtrsim 2$. However, no clustering measurements are available for Mg II absorbers at $z<0.4$ or $z \gtrsim 1$.

To test this prediction would require a large sample of galaxies and absorbers from these epochs. At $z \gtrsim 2$, a large sample of Mg II absorbers is already available (e.g. Nestor et al. 2005; Prochter et al. 2006) from searches in the Sloan Digital Sky Survey (SDSS; York et al. 2000) quasar sample (e.g. Schneider et al. 2007), but galaxy surveys that cover a cosmological volume are challenging in this redshift range. In contrast, few $\mathrm{Mg}$ II absorbers are known at $z<0.35$ where exquisite details of the galaxy population have been recorded (e.g. the SDSS), due to a lack of spectral sensitivity at wavelength $\lambda<4000 \AA$ where the low-redshift Mg II absorption features occur.

A new observing window has become available with recently commissioned UV sensitive spectrographs on the ground. In particular, the Magellan Echellette Spectrograph (MagE; Marshall et al. 2008) offers high throughput over a contiguous spectral range from $\lambda=3100 \AA$ to $1 \mu \mathrm{m}$, allowing searches for $\mathrm{Mg}$ II absorbers at redshift as low as $z=0.11$ (see also Barton \& Cooke 2009 for a similar effort at $z=0.1$ ). Building upon the existing SDSS galaxy database, we have initiated a MagE survey of Mg II absorbers in the spectra of background QSOs, whose sightlines intercept the halo of a foreground galaxy at $z \leq 0.5$. The primary goal of this project is to establish a statistically significant sample $(N \gtrsim 500)$ of low-redshift Mg II absorbers for measuring the clustering amplitude of $\mathrm{Mg}$ II absorbers at $\langle z\rangle=0.2$ based on the observed cross-correlation amplitude on co-moving scales of $1-30 \mathrm{~h}^{-1}$ Mpc. Combining the detections and non-detections in the vicinity (impact separation of $\rho \lesssim 100 \mathrm{~h}^{-1}$ physical kpc) of known galaxies will also facilitate a comprehensive study of how the properties of extended cool gas (such as the density profile and covering fraction) correlate with known stellar and ISM properties of the host galaxies. Here we introduce the MagE Mg II absorber survey and present initial results from the first year of data.

This paper is organized as follows. In Section 2, we describe the design of our MagE survey project. In Section 3 , we describe the spectroscopic observations of photometrically selected galaxies in the SDSS data archive and the MagE follow-up of quasars selected from the SDSS spectroscopic QSO catalog. We provide a summary of the data reduction and analysis procedures. In Section 4, we present the catalogs of galaxies and Mg II absorbers. In Section 5, we examine the correlation between $\mathrm{Mg}$ II absorption strength and galaxy properties. Finally, we discuss the properties of extended cool gas in galactic halos and compare our results with previous studies in Section 6. We adopt a $\Lambda$ CDM cosmology, $\Omega_{\mathrm{M}}=0.3$ and $\Omega_{\Lambda}=0.7$, with a dimensionless Hubble constant $h=H_{0} /\left(100 \mathrm{~km} \mathrm{~s}^{-1} \mathrm{Mpc}^{-1}\right)$ throughout the paper.

\section{EXPERIMENT DESIGN}

Previous surveys of $\mathrm{Mg}$ II absorbers have been carried out at $z>0.35$ (e.g. Steidel \& Sargent 1992; Nestor et al. 2005; Prochter et al. 2006), where the Mg II $\lambda \lambda 2796,2803$ doublet features are redshifted into the optical spectral window at wavelength $\lambda>3800 \AA$. Few absorbers are known at lower redshifts (Jannuzi et al. 1998; Bechtold et al. 2002). At the 
same time, wide-field surveys such as the Sloan Digital Sky Survey (SDSS; York et al. 2000) have yielded detailed maps of the large-scale galaxy distribution over 1/4 of the sky. Nearly $10^{8}$ galaxies brighter than $r^{\prime}=22$ are identified at $z<0.5$ in the SDSS archive using photometric redshift techniques. Photometric redshifts determined from the observed broad-band spectral discontinuities are found to be accurate to within an r.m.s. residual of $\left|z_{\text {phot }}-z_{\text {spec }}\right|=0.03$ for galaxies of $r^{\prime}<20$ and $\left|z_{\text {phot }}-z_{\text {spec }}\right|=0.13$ for galaxies of $r^{\prime}>20$ (e.g. Oyaizu et al. 2008). These photometrically selected galaxies, when combined with QSO absorbers along common sightlines, provide a unique opportunity for a comprehensive study of the physical origin of the absorbers and for characterizing the properties of extended gas around galaxies.

We use MagE, which offers high throughput over a contiguous spectral range from $\lambda=3100 \AA$ to $1 \mu \mathrm{m}$, to conduct a survey of $\mathrm{Mg}$ II absorbers at $z=0.11-0.35$. We aim to establish the first statistically significant sample of $\mathrm{Mg} \mathrm{II} \mathrm{ab-}$ sorbers $(N \gtrsim 500)$ at $z<0.4$, using MagE on the Magellan Clay Telescope. The primary objectives of our survey are (1) to examine the cool gas content in halos around galaxies based on the presence/absence of Mg II absorption features; (2) to study the correlation between $\mathrm{Mg}$ II absorption strength and known galaxy properties such as luminosity, impact distance, and stellar population; and (3) to quantify the origin of $\mathrm{Mg}$ II absorbers at $z \sim 0.2$ based on their large-scale clustering properties. The results, when combined with known statistical properties of $\mathrm{Mg}$ II absorbers at $z=0.4-1$, will allow us to establish an empirical characterization of the evolution of cool baryons in dark matter halos (e.g. Tinker \& Chen 2010).

To maximize the efficiency of the $\mathrm{Mg}$ II absorber search, we select those QSO sightlines that are close to a foreground galaxy with photometric redshift $z_{\text {phot }} \leq 0.4$. The maximum angular separation for the selected QSO-galaxy pairs is determined based on previous observations of extended $\mathrm{Mg}$ II absorbing gas around intermediate-redshift galaxies. Using 23 galaxy-Mg II absorber pairs at $z=0.207-0.892$, Chen \& Tinker (2008) found a distinct boundary at $\rho=\hat{R}_{\text {gas }}$, beyond which no $\mathrm{Mg}$ II absorbers with rest-frame absorption equivalent width $W_{r}(2796)>0.01 \AA$ are found. At $\rho<\hat{R}_{\text {gas }}$, the incidence of $\mathrm{Mg}$ II absorbers was found to be $\approx 80-86 \%$. In addition, the observed correlation between $W_{r}(2796)$ and the physical projected distance $\rho$ to the galaxy is well described by an isothermal density profile with $\hat{R}_{\text {gas }}$ scaled by galaxy $B$-band luminosity $\left(L_{B}\right)$ or absolute $B$-band magnitude $\left(M_{B}\right)$ according to

$$
\frac{\hat{R}_{\text {gas }}}{\hat{R}_{\text {gas } *}}=\left(\frac{L_{B}}{L_{B_{*}}}\right)^{0.35}=10^{0.14\left(M_{B}-M_{B_{*}}\right)}
$$

and

$$
\hat{R}_{\text {gas } *}=91 h^{-1} \mathrm{kpc} .
$$

We select galaxies from the SDSS Data Release 6 (DR6) archive, and adopt Equations (1) and (2) as the fiducial model for calculating the expected gaseous radii for galaxies in the SDSS Data Release 6 (DR6) archive. The rest-frame absolute $B$-band magnitude of each galaxy is estimated using the bestfit photometric redshift $z_{\text {phot }}$ from Oyaizu et al. (2008) ${ }^{5}$ and its

\footnotetext{
5 We have adopted the photometric redshifts evaluated under case 'cc2' in Oyaizu et al. (2008), which included four colors $u^{\prime}-g^{\prime}, g^{\prime}-r^{\prime}, r^{\prime}-i^{\prime}$, $i^{\prime}-z^{\prime}$, and three concentration indices in the $g^{\prime} r^{\prime} i^{\prime}$ bands. According to these authors, 'cc2' provides the best and most realistic redshift estimates.
}

model $g^{\prime}$-band magnitude from the SDSS archive ${ }^{6}$, following

$$
M_{B}=g^{\prime}-5 \log \frac{D_{L}}{10 \mathrm{pc}}+2.5 \log (1+z)-k(z),
$$

where $k(z)$ is the $k$-correction term to account for the color difference between the observed $g^{\prime}$ band and corresponding restframe $B$ band. We estimate the $k$-correction of each galaxy using an $S_{\text {bc }}$ galaxy template from Coleman et al. (1980), and adopt $M_{B_{*}}-5 \log h=-19.8$ from Faber et al. (2007) when computing $\hat{R}_{\text {gas }}$ using Equations (1) and (2).

From a cross-comparison between the SDSS DR6 catalogs of galaxies and QSOs (Adelman-McCarthy et al. 2008), we identify QSO and galaxy pairs according to the following criteria. First, we consider galaxies at $z_{\text {phot }} \leq 0.4$ to explore the unique spectral window offered by MagE for the Mg II absorber search. We consider QSOs that are brighter than $u^{\prime}=19.5$ in order to obtain sufficient $S / N$ in a relatively short exposure using MagE on the Magellan Clay Telescope. We select QSOs at $z \leq 1.5$ to avoid contamination due to the Ly $\alpha$ forest. Next, we impose additional constraints to select only QSOs at $z>0.4$ and at more than $10,000 \mathrm{~km} \mathrm{~s}^{-1}$ behind the galaxy in the redshift space, in order to minimize the incidence of correlated QSO and galaxy pairs (e.g. Wild et al. 2008). Finally, we select QSO-galaxy pairs with projected separations less than $\hat{R}_{\text {gas }}$ from Equations (1) and (2). This procedure yields $\approx 3900$ close QSO-galaxy pairs at Declination $<+20^{\circ}$ with angular separation $\theta<180^{\prime \prime}$ on the sky.

The galaxies in the pair sample span a broad range in intrinsic colors, luminosity, and impact separation. Although they are pre-selected to occur within the fiducial radius of $\rho=\hat{R}_{\text {gas }}$, uncertainties in the photometric redshifts allow us to study the gaseous halo in regions beyond $\hat{R}_{\text {gas }}$.

\section{OBSERVATIONS AND DATA REDUCTION}

Our program requires spectroscopic data of both galaxies and absorbers along common lines of sight toward background quasars. Here we describe the observation and data reduction procedures.

\subsection{Galaxy Spectroscopy}

We have obtained optical spectra of 89 photometrically selected SDSS galaxies, using the Double Imaging Spectrograph (DIS; Lupton et al. 1995) on the $3.5 \mathrm{~m}$ telescope at the Apache Point Observatory and MagE (Marshall et al. 2008) on the Magellan Clay Telescope at the Las Campanas Observatory. All of these galaxies satisfy the selection criteria described in $\S 2$. The medium-to-high resolution spectra allow us to obtain precise and accurate redshift measurements of these galaxies, which are necessary for establishing (or otherwise) a physical connection with absorbers found along nearby QSO sightlines. The spectroscopic observations of these SDSS galaxies are summarized here.

Long-slit spectra of 41 galaxies were obtained using DIS over the period from October 2008 through June 2009. The blue and red cameras have a pixel scale of $0.4^{\prime \prime}$ and $0.42^{\prime \prime}$ per pixel, respectively, on the $3.5 \mathrm{~m}$ telescope. We used a $1.5^{\prime \prime}$ slit, the B400 grating in the blue channel for a dispersion of

\footnotetext{
6 All object magnitudes quoted in this paper are in the $A B$ system.. To remind the readers, the SDSS imaging survey reaches a 5- $\sigma$ limiting magnitude of $A B=22 \mathrm{mag}$ in the $u^{\prime}, g^{\prime}$, and $r^{\prime}$ bands, and $A B \approx 21 \mathrm{mag}$ in the $i^{\prime}$ and $z^{\prime}$ bands (York et al. 2000). In selecting our galaxies, we do not require a detection in all five bands. At a limiting magnitude of $r^{\prime}=22$, we will be able to uncover $L_{*}$ galaxies at $z=0.4$ and sub- $L_{*}$ galaxies at lower redshifts.
} 


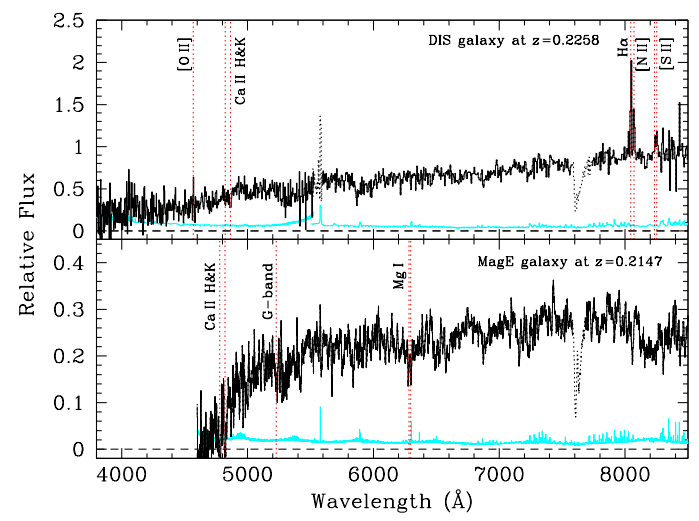

FIG. 1.- Top: An example DIS spectrum of a galaxy (SDSSJ130555.49+014928.62) at $z=0.2258$. Bottom: An example MagE spectrum of a galaxy (SDSSJ230845.53-091445.97) at $z=0.2147$. Contaminating sky features are dotted out. The corresponding $1-\sigma$ error spectrum is shown at the bottom of each panel.

$1.83 \AA$ A per pixel, and the R300 grating in the red channel for a dispersion of 2.31 A per pixel. The blue and red channels with the medium-resolution gratings together offer contiguous spectral coverage from $\lambda=3800 \AA$ to $\lambda=9800 \AA$ and a spectral resolution of FWHM $\approx 500 \mathrm{~km} \mathrm{~s}^{-1}$ at $\lambda=4400 \AA$ and $\mathrm{FWHM} \approx 400 \mathrm{kms}^{-1}$ at $\lambda=7500 \AA$. The observations were carried out in a series of two to three exposures of between $1200 \mathrm{~s}$ and $1800 \mathrm{~s}$ each, and no dither was applied between individual exposures. Flat-field frames were taken in the afternoon prior to the beginning of each night. Calibration frames for wavelength solutions were taken immediately after each set of science exposures using the truss lamps on the secondary cage. When the sky was clear, we also observed a spectrophotometric standard star observed at the beginning of each night for relative flux calibration.

All of the DIS spectroscopic data were reduced using standard long-slit spectral reduction procedures. The spectra were calibrated to vacuum wavelengths, corrected for the heliocentric motion, and flux-calibrated using a sensitivity function derived from observations of a flux standard. Redshifts of the galaxies were determined based on a cross-correlation analysis with a linear combination of SDSS eigen spectra of galaxies. The typical redshift uncertainty was found to be $\Delta z \sim 0.0003$. An example of the DIS galaxy spectra is presented in the top panel of Figure 1.

Optical spectra of 48 galaxies were obtained using MagE in September 2008, February 2009, March 2009, and June 2009 . The camera has a plate scale of $0.3^{\prime \prime}$ per pixel. We used a $1^{\prime \prime}$ slit and $2 \times 1$ binning during readout, which yielded a spectral resolution of FWHM $\approx 150 \mathrm{~km} \mathrm{~s}^{-1}$. Depending on the brightness of the galaxies, the observations were carried out in a sequence of one to two exposures of duration $300 \mathrm{~s}$ to $1200 \mathrm{~s}$ each. The galaxy data were processed and reduced using data reduction software that was originally developed by G. Becker and later modified by us to work with binned spectral frames. Wavelengths were calibrated using a ThAr frame obtained immediately after each exposure and subsequently corrected to vacuum and heliocentric wavelengths. Flux calibration was performed using a sensitivity function

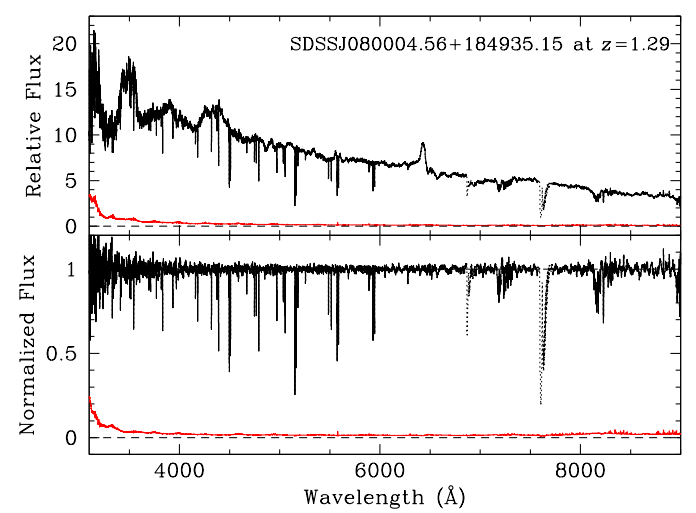

FIG. 2.- Top: An order-combined MagE spectrum of SDSSJ080004.56+184935.15 at $z=1.29$. Bottom: Continuum normalized, stacked spectrum of the same QSO. Absorption features due to the Atmosphere are dotted out. This sightline displays multiple strong Mg II absorbers at $z=0.254-1.12$, including one at $z=0.254$ at $\rho=22.2 h^{-1} \mathrm{kpc}$ and $\Delta v=-191 \mathrm{~km} \mathrm{~s}^{-1}$ from a known galaxy. The corresponding 1- $\sigma$ error spectrum is shown at the bottom of each panel.

derived from observations of the flux standard GD50. Individual flux-calibrated orders were coadded to form a single spectrum that covers a spectral range from $\lambda \approx 4000 \AA$ to $\lambda=9600 \AA$ A Redshifts of the galaxies were determined based on a cross-correlation analysis with a linear combination of SDSS eigen spectra of galaxies. The typical redshift uncertainty was found to be $\Delta z \sim 0.0001$. An example of the MagE galaxy spectra is presented in the bottom panel of Figure 1.

We have also located five additional galaxies, which satisfy the selection criteria described in $\S 2$ and already have optical spectra and robust spectroscopic redshifts available in the public SDSS data archive. These galaxies are included in our final galaxy sample for the search of $\mathrm{Mg}$ II absorbers. A journal of the spectroscopic observations of the full galaxy sample is in Table 1.

\subsection{Echellette Spectra of QSOs}

Echellette spectroscopic observations of 70 QSOs were obtained using the MagE spectrograph (Marshall et al. 2008) on the Magellan Clay telescope over the period from January 2008 through June 2009. We used a $1^{\prime \prime}$ slit for the majority of the QSOs (with a few sources taken with a $0.7^{\prime \prime}$ slit) and $1 \times 1$ binning during readout, which yielded a spectral resolution of FWHM $\approx 70 \mathrm{~km} \mathrm{~s}^{-1}$ ( or FWHM $\approx 50 \mathrm{~km} \mathrm{~s}^{-1}$ for spectra taken with a $0.7^{\prime \prime}$ slit). Depending on the brightness of the QSOs, the observations were carried out in a sequence of one to three exposures of duration $600 \mathrm{~s}$ to $2400 \mathrm{~s}$ each. The QSO spectra were processed and reduced using a reduction pipeline developed by G. Becker and kindly offered to us by the author. Wavelengths were calibrated using a ThAr frame obtained immediately after each exposure and subsequently corrected to vacuum and heliocentric wavelengths. Flux calibration was performed using a sensitivity function derived from observations of the flux standard GD50. Individual flux calibrated echellette orders were coadded to form a single spectrum that covers a spectral range from $\lambda=3050 \AA$ to $\lambda=1 \mu \mathrm{m}$. These order-combined individual exposures were then continuum normalized and stacked to form one final combined 

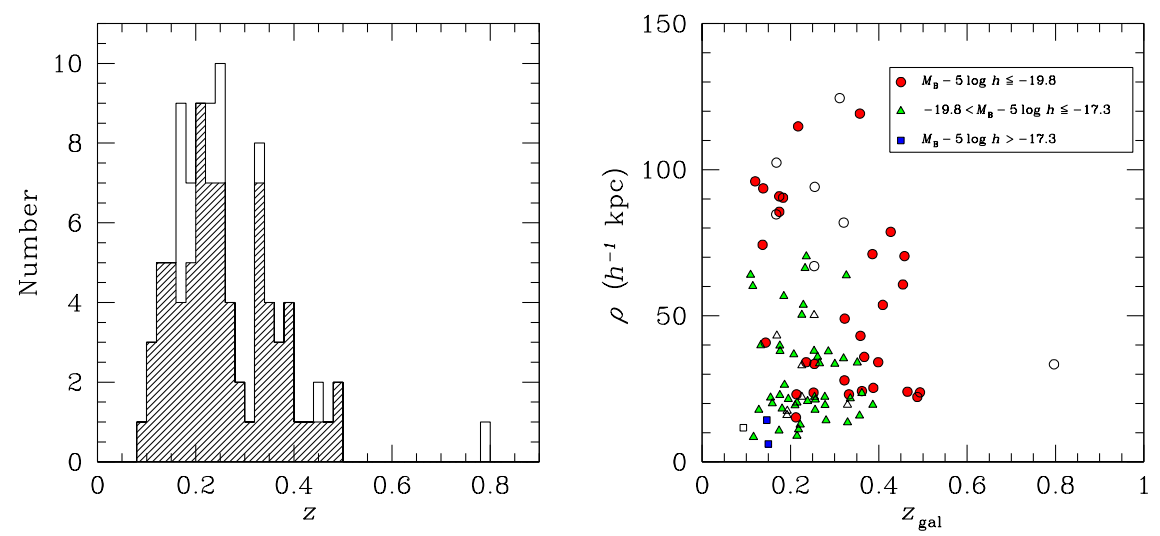

FIG. 3.- Left: Redshift distritbutions of 94 galaxies in the 'full' sample (solid histogram) and of 77 galaxies in the 'isolated' galaxy sample (shaded histogram). Right: Distribution of projected separations between the spectroscopically confirmed galaxies and their nearby QSO sightlines. Galaxies of $B$-band luminosity $L_{B}>L_{B_{*}}$ are shown in circles; galaxies of $L_{B}=(0.1-1) L_{B_{*}}$ in triangles; and galaxies of $L_{B}<0.1 L_{B_{*}}$ in squares.

spectrum per QSO using an optimal weighting routine. The continuum was determined using a low-order polynomial fit to spectral regions that are free of strong absorption features. The flux calibrated spectra have $S / N \gtrsim 10$ per resolution element across the entire spectral range. Examples of an ordercombined spectrum and a continuum-normalized final stack are shown in Figure 2. A journal of the MagE QSO observations is in Table 2.

\section{THE GALAXY AND MG II ABSORBER CATALOGS}

\subsection{The Galaxy Sample}

We have established a sample of 94 galaxies with $r^{\prime}<22.3$ and robust spectroscopic redshifts at $z<0.8$ in fields around 70 distant background QSOs (zoso $>0.6$ ). These 94 spectroscopically confirmed galaxies form the 'full' sample of our study. Within the full sample, 17 galaxies are found with at least one close neighbor at $\rho<\hat{R}_{\text {gas }}$ and velocity separation $\Delta v<300 \mathrm{~km} \mathrm{~s}^{-1}$, including one galaxy that occurs in the vicinity of a background QSO at $z \approx 0.8$. The presence of a close neighbor implies that the galaxy is likely to reside in a group environment. The association between absorbers and individual galaxies becomes uncertain in a group environment. In addition, interactions between group members are expected to alter the properties of gaseous halos (e.g. Gunn \& Gott 1972; Balogh et al. 2000; Verdes-Montenegro et al. 2001). Therefore, these galaxies form a 'group'-galaxy subsample and are considered separately from the remaining 'isolated'-galaxy subsample in our analysis below. Here we summarize the galaxy properties in the full sample.

We first examine the redshift distribution of the galaxies. Figure 3 shows in the left panel the redshift distribution of all 94 spectroscopically confirmed galaxies in our survey (full histogram) and the redshift distribution of the 'isolated' galaxy subsample (shaded histogram). Figure 4 shows the comparison of photometric redshifts $z_{\text {phot }}$ and spectroscopic redshift $z_{\text {spec }}$ for galaxies in our full sample. Excluding the single outlier at $z \approx 0.8$, we find that SDSS photometric redshifts for galaxies brighter than $r^{\prime}=21.6$ are accurate to within a mean residual of $\left\langle\left(z_{\text {phot }}-z_{\text {spec }}\right) /\left(1+z_{\text {spec }}\right)\right\rangle=0.01$ and a corresponding r.m.s. scatter of $\delta z \equiv \Delta z /(1+z)=0.06$. There exists an increased uncertainty in the photometric redshifts for galaxies at $z \gtrsim 0.35$, resulting in the apparent 'tilt' seen in the left panel. We also examine photometric redshift uncertainties versus galaxy brightness and intrinsic color. The right panel of Figure 4 shows that the precision and accuracy of photometric redshifts are still higher for brighter $\left(r^{\prime}<20\right)$ and redder galaxies (with rest-frame $B_{A B}-R_{A B}$ color consistent with elliptical/S0 galaxies; see Equation 4 and Figure 5 for the classification of different galaxy types). With the exception of one outlier at $z \approx 0.8$, the target selection based on known photometric redshifts has allowed us to effectively identify foreground galaxies at $z=0.1-0.4$ that occur at close projected distances to the line of sight toward a background QSO.

Next, we present the impact parameter distribution of the galaxies to a QSO sightline in the right panel of Figure 3. 'Group' galaxies are displayed in open symbols and 'isolated' galaxies in solid symbols. Galaxies in different luminosity ranges are shown in different shapes of symbols. Figure 3 shows that luminous galaxies with $B$-band luminosity $L_{B}>L_{B_{*}}$ (circles) $^{7}$ span a range in their projected distance to a QSO sightline from $\rho=15.2 h^{-1} \mathrm{kpc}$ to $\rho=119.2 h^{-1} \mathrm{kpc}$, while sub- $L_{*}$ galaxies of $L_{B}=(0.1-1) L_{B_{*}}$ (triangles) cover a range from $\rho=8.5 h^{-1} \mathrm{kpc}$ to $\rho=70.3 h^{-1} \mathrm{kpc}$. Our sample also includes two faint dwarfs with $L_{B}<0.1 L_{B_{*}}$ (squares) at $\rho<15 h^{-1} \mathrm{kpc}$. In addition, the QSO point spread function prevents us from finding galaxies at angular distances $\Delta \theta \lesssim 3^{\prime \prime}$ from the QSO sightlines in the SDSS catalog. The minimum project distances we can probe using the SDSS galaxy sample changes from $\rho_{\min }<10 h^{-1} \mathrm{kpc}$ at $z \lesssim 0.15$ to $\rho_{\min }>15 h^{-1} \mathrm{kpc}$ at $z \gtrsim 0.4$. The distribution shows that our survey is designed to study halo gas (rather than the interstellar medium) of distant galaxies. Our sample includes more luminous galaxies (presumably more massive and therefore residing in more extended gaseous halos) at larger impact parameters with the maximum survey radius determined by the fiducial model $(\S 2)$.

The rest-frame absolute $B$-band magnitudes and rest-frame $B-R$ colors of all 94 galaxies are calculated based on the spectroscopic redshifts. To carry out this calculation, we first

${ }^{7}$ We adopt $M_{B_{*}}-5 \log h=-19.8$ from Faber et al. (2007), which best characterizes the blue galaxy population at $z \sim 0.4$. 

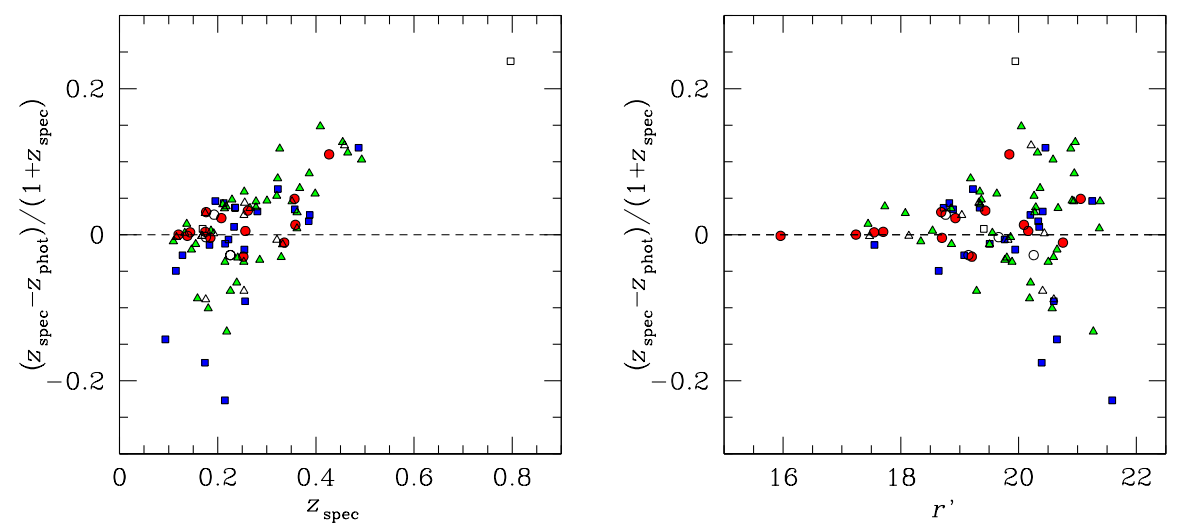

FIG. 4.- Comparisons of photometric redshifts $z_{\text {phot }}$ and spectroscopic redshift $z_{\text {spec }}$ for galaxies at different redshift (left panel) and of different $r^{\prime}$-band magnitude (right panel). Galaxies with intrinsic $B_{A B}-R_{A B}$ color consistent with elliptical/S0 galaxies are shown in circles; disk galaxies are shown in triangles; and irregular/star-forming galaxies are shown in squares (see Equation 4 and Figure 5 for the classification of different galaxy types). Galaxies in the 'group' subsample are shown in open symbols.

adopt the best-fit model magnitudes from the SDSS photometric catalog and compute the composite model magnitudes in the $u^{\prime} g^{\prime} r^{\prime} i^{\prime} z^{\prime}$ bandpasses following the instructions of Scranton et al. $(2005)^{8}$. Then, the composite model magnitudes are corrected for the Galactic extinctions following Schlegel et al. (1998). Finally for each galaxy, we identify the bandpasses that correspond most closely to the rest-frame $B$ and $R$ bands based on its spectroscopic redshift. We interpolate between the composite model magnitudes to determine the intrinsic $B-R$ color and the $k$-term necessary to correct the remaining color difference for deriving the rest-frame absolute $B$-band magnitude following Equation (3).

Figure 5 shows the redshift distribution of rest-frame absolute $B$-band magnitudes of our galaxies, spanning a range from $M_{B}-5 \log h=-16.22$ to $M_{B}-5 \log h=-22.5$. We also examine the underlying stellar population by comparing the observed optical colors with expectations from known spectral energy distribution templates of galaxies of different morphological type. We consider the E/S0, Sbc, Scd, and Irr galaxy templates from Coleman et al. (1980) and calculate the expected colors versus redshift under a no-evolution assumption (see also Fukugita et al. 1995). The right panel of Figure 5 shows that the optical colors of our galaxies are in broad agreement with the expected colors of galaxies across a broad range of morphology. We classify the galaxies based on the intrinsic $B_{A B}-R_{A B}$ color according to the following criteria:

$$
\begin{array}{ll}
B_{A B}-R_{A B}>1.1 & \text { elliptical/S0, } \\
0.6<B_{A B}-R_{A B} \leq 1.1 & \text { disk galaxies, } \\
B_{A B}-R_{A B}<0.6 & \text { irregular/star-forming galaxies.(4) }
\end{array}
$$

Of the 94 galaxies in our spectroscopic sample, 18 have the intrinsic $B_{A B}-R_{A B}$ color consistent with an elliptical or S0 galaxy, 52 are consistent with a disk galaxy, and 24 are consistent with an irregular or younger galaxies. The classification is qualitatively consistent with the spectral features seen

\footnotetext{
8 The composite model magnitude is evaluated based on the composite flux, $f_{\text {composite }}$, which is a linear combination of the best-fit fluxes contained in an exponential profile $f_{\exp }$ and a de Vaucouleurs profile $f_{\mathrm{deV}}$ : $f_{\text {composite }}=f r a c_{\mathrm{deV}} f_{\mathrm{deV}}+\left(1-f r a c_{\mathrm{deV}}\right) f_{\text {exp }}$. The composite model magnitude is considered to provide the best-estimate of the total flux of a galaxy (see Scranton et al. 2005 for more detailed discussion).
}

in the galaxy spectra. Of the 18 galaxies classified as elliptical or S0, more than $2 / 3$ display spectral features dominated by absorption transitions due to Ca II H\&K and G-band. A more detailed analysis is presented in a separate paper (Helsby et al. in preparation).

\subsection{The Galaxy-Mg II Absorber Pair Sample}

To establish a galaxy-Mg II absorber pair sample for the subsequent analysis, we examine the echellette spectra of the background QSOs and search for the corresponding Mg II absorption doublet at the locations of the galaxies presented in $\S 4.1$. Specifically, we first search for the corresponding doublet features within velocity separation $\Delta v= \pm 1000 \mathrm{~km} \mathrm{~s}^{-1}$ of the galaxy redshifts. Then we accept absorption lines according to a $2 \sigma$ detection threshold criterion, which is appropriate because the measurements are performed at known galaxy redshifts. Next, we determine the absorber redshift based on the best-fit line centroid of a Gaussian profile analysis of the doublets. Uncertainties in the absorber redshifts are less than $\delta v=\left(c \Delta z /(1+z)=25 \mathrm{~km} \mathrm{~s}^{-1}\right.$. Next, we measure 2- $\sigma$ upper limits to the 2796- $\AA$ absorption equivalent widths for galaxies that are not paired with corresponding absorbers.

The procedure identifies $47 \mathrm{Mg}$ II absorbers and 24 upper limits in the vicinities of 71 isolated galaxies, and seven Mg II absorbers and one upper limit around 17 'group' galaxies. We are unable to obtain significant constraints for the presence/absence of $\mathrm{Mg}$ II absorbers around five galaxies, because the expected Mg II absorption features are blended with either strong C IV $\lambda \lambda 1548,1550$ absorption features at higher redshifts or the atmosphere $\mathrm{O} 3$ absorption complex at $\lambda \approx 3200$. Finally, one galaxy is found at $z_{\mathrm{spec}}=0.0934$, outside of the redshift range targeted for the MagE Mg II absorber survey. Examples of the galaxy-Mg II absorber pairs, including upper limits, are presented in Figure 6. A summary of the properties of the spectroscopically confirmed galaxies is presented in Table 3, where we list for each galaxy in columns (1)(8) the ID, Right Ascension and Declination offsets from the QSO $\Delta \alpha$ and $\Delta \delta$, redshift $z_{\text {gal }}$, impact parameter $\rho$, apparent $r^{\prime}$-band magnitude, $B_{A B}-R_{A B}$ color, and absolute $B$-band magnitude $M_{B}-5 \log h$. Measurement uncertainties in $r^{\prime}$ and $M_{B}-5 \log h$ are typically 0.05 dex. 

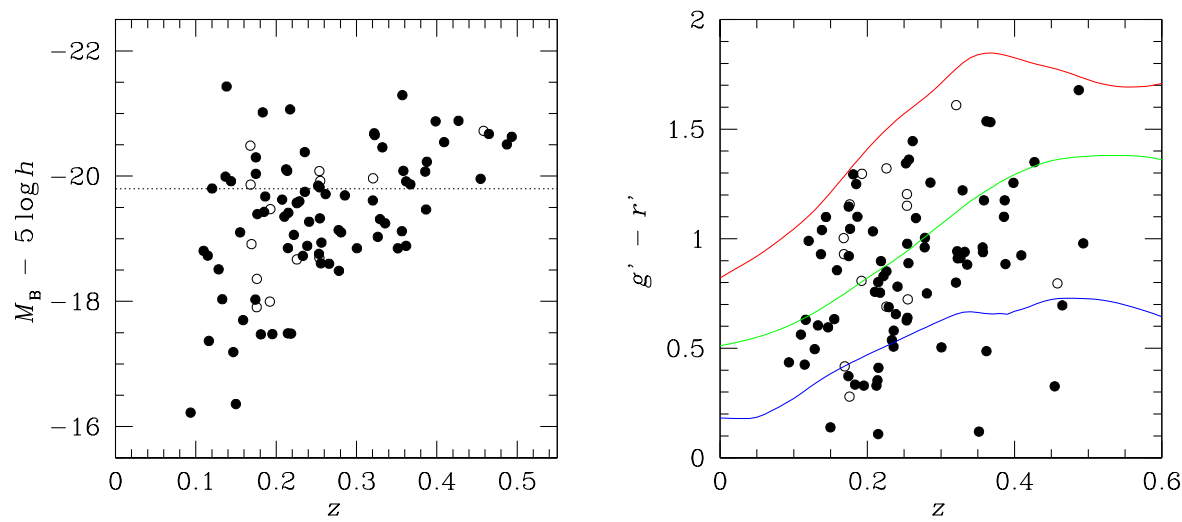

FIG. 5.- Redshift distributions of rest-frame absolute $B$-band magnitudes (left) and observed $g^{\prime}-r^{\prime}$ colors (right) of the spectroscopically confirmed galaxies in the 'group' subsample (open histograms/symbols) and in the 'isolated' subsample (shaded histograms/closed symbols). The galaxies span a range in the rest-frame absolute $B$-band magnitude from $M_{B}-5 \log h=-16.22$ to $M_{B}-5 \log h=-22.5$, and a broad range in intrinsic colors. The dotted line in the left panels marks $M_{B_{*}}$ from Faber et al. (2007). The solid curves in the right panel indicate the expected $g^{\prime}-r^{\prime}$ color versus redshift based on non-evolving galaxy templates for E/S0 (top curve), Sbc (middle curve), and irregular (bottom curve) galaxies from Coleman et al. (1980).

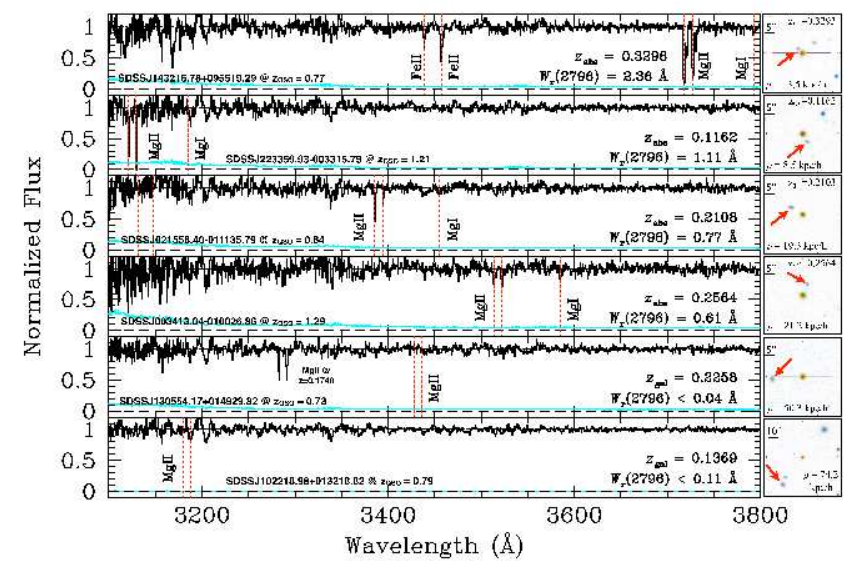

FIG. 6.- Examples of the galaxy-Mg II absorber pairs in our sample. For each field, we present the QSO spectrum in the left panel and the corresponding SDSS image centered at the QSO in the right panel. The galaxies in our survey with confirmed spectroscopic redshifts is indicated by the arrow. The projected distance to the QSO is shown in the lower right corner of each image panel. The absorption features corresponding to the redshifts of the galaxies are indicated by dotted lines in the left panels. The absorber redshift is shown in the lower right corner of each spectrum. For non-detections in the bottom two panels, we note the redshift of the galaxy and the $2-\sigma$ upper limit of $W_{r}(2796)$.

Impact parameter separations of the galaxy and absorber pairs in the 'isolated' sample range from $\rho=6.1$ to $119.2 \mathrm{~h}^{-1}$ physical $\mathrm{kpc}$ with a median of $\langle\rho\rangle_{\text {med }}=27.9 h^{-1} \mathrm{kpc}$. The redshifts of the 'isolated' galaxies range from $z=0.1097$ to $z=0.4933$ with a median of $\langle z\rangle_{\text {med }}=0.2533$. The rest-frame absolute $B$-band magnitudes of the galaxies span a range from $M_{B}-5 \log h=-16.4$ to $M_{B}-5 \log h=-21.4$ with a median of $\left\langle M_{B}-5 \log h\right\rangle=-19.6$. The rest-frame $B_{A B}-R_{A B}$ colors range from $B_{A B}-R_{A B} \approx 0$ to $B_{A B}-R_{A B} \approx 1.5$ with a median of $\left\langle B_{A B}-R_{A B}\right\rangle_{\text {med }} \approx 0.8$.

The redshift and absorption equivalent width of the corresponding Mg II absorption feature for each of the 71 galaxies in our sample are listed in columns (9)-(10) of Table 3. Figure 7 shows the relative velocity distribution of the 47 galaxy-Mg II absorber pairs in the isolated sample. We find that with the exception of one pair, which exhibits a velocity separation of $v_{\mathrm{Mg} \text { II-Galaxy }}=-645 \mathrm{~km} \mathrm{~s}^{-1}$, the velocity separation between the Mg II absorbers and their member galaxies is well characterized by a Gaussian distribution of mean velocity difference $\left\langle v_{\text {Mg II-Galaxy }}\right\rangle=16 \mathrm{~km} \mathrm{~s}^{-1}$ and dispersion $\sigma=137$ $\mathrm{km} \mathrm{s}^{-1}$. The velocity dispersion between the galaxy-Mg II absorber pair sample is comparable to the velocity dispersion seen in a Milky-Way size galaxy (see also Steidel et al. 2002; Chen et al. 2005; Kacprzak et al. 2010 for rotation curve comparisons of galaxies and strong absorbers at intermediate redshifts), supporting a physical association between the absorbing gas and the member galaxy. 


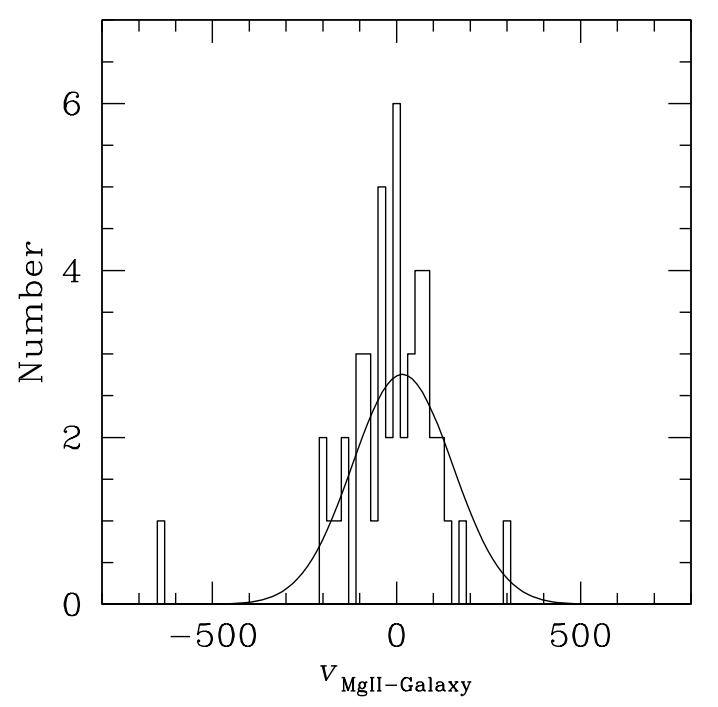

FIG. 7.- Relative velocity Distribution of the 47 galaxy-Mg II absorber pairs in the 'isolated' sample. The pair sample is well characterized by a Gaussian distribution (the solid curve) with a mean velocity difference of $\left\langle v_{\text {Mg II-Galaxy }}\right\rangle=16 \mathrm{~km} \mathrm{~s}^{-1}$ and dispersion of $\sigma=137 \mathrm{~km} \mathrm{~s}^{-1}$.

We examine the correlation between galaxies and $\mathrm{Mg}$ II absorbers identified along common lines of sight, using the sample of 47 galaxy-Mg II absorber pairs and 24 galaxies at $\rho \lesssim 120 h^{-1} \mathrm{kpc}$ that do not give rise to $\mathrm{Mg}$ II absorption to a sensitive upper limit. Figure 8 shows the distribution of $W_{r}(2796)$ versus $\rho$ for our 'isolated' sample of 71 galaxies. Galaxies with intrinsic $B_{A B}-R_{A B}$ color consistent with elliptical/S0 galaxies are shown in circles, those consistent with disk galaxies are shown in triangles; and irregular/starforming in squares. Points with arrows in Figure 8 indicate $2 \sigma$ upper limits. Galaxies in the 'group' subsample are also included as open symbols for comparison. Similar to previous results (e.g. Lanzetta \& Bowen 1990; Kacprzak et al. 2008), we find that on average $W_{r}(2796)$ declines with increasing $\rho$ for galaxies in an 'isolated' environment. In contrast, while galaxies from a 'group' environment appear to occupy a similar $W_{r}(2796)$ versus $\rho$ space with 'isolated' galaxies, they do not exhibit a strong inverse correlation. In this section, we describe the methods we use for obtaining and assessing the best-fit models that represent the data and present the results.

\subsection{Method}

To obtain the best-fit model that characterizes the correlation between the strength of Mg II absorbers and the properties of galaxies, we adopt a parameterized functional form

$$
y=f(x 1, x 2, . .)
$$

where $y$ is the predicted absorber strength and is always $\log W_{r}(2796)$ in our analysis, and $x_{i}$ 's are independent measurements of galaxy properties such as galaxy impact parameter $\rho$, rest-frame absolute magnitude $M_{B}-5 \log h$, redshift $z$, or intrinsic color $B_{A B}-R_{A B}$. We consider a simple power-law model for characterizing the $W_{r}(2796)$ versus $\rho$ relation. In logarithmic space, the model is expressed as a linear function according to

$$
\log \bar{W}_{r}^{\mathrm{p}}(2796)=a_{0}+a_{1} \log \rho+a_{2}\left(M_{B}-M_{B_{*}}\right)+\ldots
$$

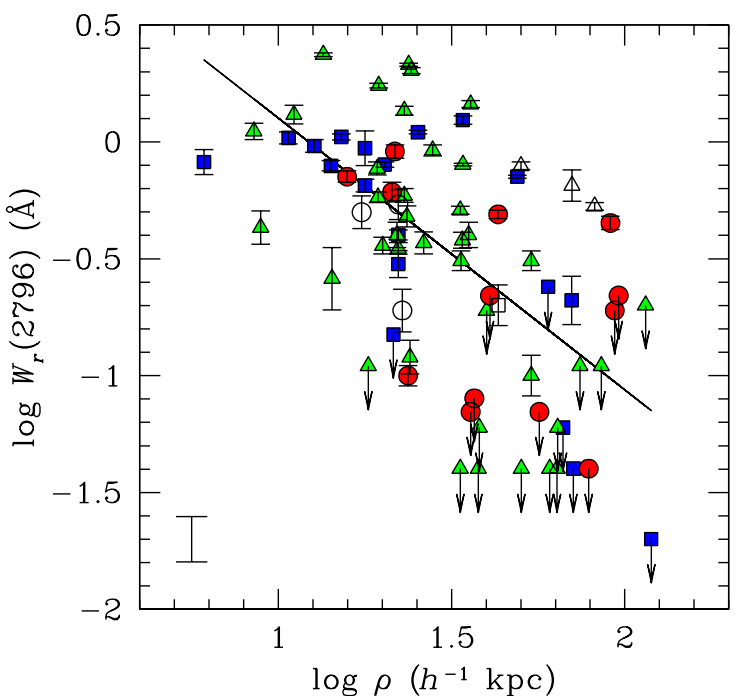

FIG. 8.- Comparison of the corresponding rest-frame absorption equivalent width $W_{r}(2796)$ versus galaxy impact parameter $\rho$ for a sample of 71 galaxies. Circles represent galaxies with intrinsic $B_{A B}-R_{A B}$ colors consistent with elliptical or S0 galaxies, triangles represent early- and late-type disk galaxies, and squares represent irregular, star-forming galaxies. Points with arrows indicate $2 \sigma$ upper limits. The solid line indicate the best-fit power-law model. The errorbar in the lower-left corner indicates the intrinsic scatter estimated based on the likelihood analysis discussed in $\$ 5.1$ and Equation (7). We also include galaxies that are in the 'group' subsample (open symbols) for comparisons.

To determine the values of different coefficients $a_{i}$ 's, we perform a maximum-likelihood analysis that includes the upper limits in our galaxy and Mg II absorber pair sample. The likelihood function of this analysis is defined as

$$
\begin{aligned}
\mathcal{L}(\bar{y})= & \left(\prod_{i=1}^{n} \exp \left\{-\frac{1}{2}\left[\frac{y_{i}-\bar{y}\left(\rho_{i}, L_{B_{i}}\right)}{\sigma_{i}}\right]^{2}\right\}\right) \times \\
& \left(\prod_{i=1}^{m} \int_{y_{i}}^{-\infty} d y^{\prime} \exp \left\{-\frac{1}{2}\left[\frac{y^{\prime}-\bar{y}\left(\rho_{i}, L_{B_{i}}\right)}{\sigma_{i}}\right]^{2}\right\}\right),
\end{aligned}
$$

where $y_{i}=\log W_{i}$ is the observed value of galaxy $i, \bar{y}=$ $\log \bar{W}_{r}(2796)$ is the model expectation, and $\sigma_{i}$ is the measurement uncertainty of $y_{i}$. The first product of Equation (7) extends over the $n$ measurements and the second product extends over the $m$ upper limits. (This definition of the likelihood function is appropriate if the residuals about the mean relationship are normally distributed.) We also consider the possibility that $\sigma_{i}$ includes a significant intrinsic scatter (which presumably arises due to intrinsic variations between individual galaxies) as well as measurement error. We express $\sigma_{i}$ as a quadratic sum of the cosmic scatter $\sigma_{c}$ and the measurement error $\sigma_{m_{i}}$

$$
\sigma_{i}^{2}=\sigma_{c}^{2}+\sigma_{m_{i}}^{2}
$$

where the intrinsic scatter is defined by

$\sigma_{c}^{2}=\operatorname{med}\left(\left\{y_{i}-\bar{y}\left(\rho_{i}, L_{B_{i}}, \ldots\right)-\frac{1}{n} \sum_{j=1}^{n}\left[y_{j}-\bar{y}\left(\rho_{j}, L_{B_{j}}, \ldots\right)\right]\right\}^{2}-\sigma_{m_{i}}^{2}\right)$. 
Because $\sigma_{c}$ depends on the maximum-likelihood solution $\bar{y}=$ $\log \bar{W}\left(\rho_{i}, L_{B_{i}}\right)$, the maximum-likelihood solution is obtained iteratively with respect to equations (7) and (9).

\subsection{Dependence of Extended Gas on Galaxy Impact Parameter and B-band Luminosity}

Adopting a power-law function for describing the $W_{r}(2796)$ versus $\rho$ distribution in Figure 8, we find based on the maximum-likelihood analysis a best-fit model of

$$
\log \bar{W}_{r}^{\mathrm{p}}(2796)=-(1.17 \pm 0.10) \log \rho+(1.28 \pm 0.13)
$$

with an intrinsic scatter of $\sigma_{c}=0.195$. The r.m.s. residual between the observed and model $\mathrm{Mg}$ II absorber strengths is found to be r.m.s. $\left(\log W_{r}-\log \bar{W}\right)=0.350$. Errors associated with the best-fit coefficients are 1- $\sigma$ uncertainties. We present the best-fit model and the intrinsic scatter in Figure 8.

The results of the likelihood analysis demonstrates at a high confidence level that the Mg II absorber strength $W_{r}(2796)$ scales inversely with galaxy impact parameter. To assess the significance of this anti-correlation, we perform a generalized Kendall test (Feigelson \& Nelson 1985) that accounts for the presence of non-detections and find that the observed distribution of $W_{r}(2796)$ versus $\rho$ deviates from a random distribution at more than 5.5- $\sigma$ level of significance.

Despite a statistically significant anti-correlation seen between $W_{r}(2796)$ and $\rho$, there exists a large scatter between the data and the best-fit power-law model (Equation 10). Previous authors (Chen \& Tinker 2008; Kacprzak et al. 2008) have shown that the observed Mg II absorber strength scales with galaxy $B$-band luminosity. Recall that our galaxy sample spans a range in rest-frame absolute $B$-band magnitude from $M_{B}-5 \log h=-16.4$ to $M_{B}-5 \log h=-21.4$. Here we examine whether including intrinsic $B$-band luminosity helps to further strengthen this anti-correlation.

Adopting a power-law function to parameterize the scaling relation, we find based on the likelihood analysis that the observed absorber strength is best described by $\log W_{r}(2796)=$ $-(1.52 \pm 0.10) \log \rho-(0.20 \pm 0.03)\left(M_{B}-M_{B_{*}}\right)+(1.88 \pm 0.15)$ (dotted line in Figure 9), with an intrinsic scatter of $\sigma_{c}=$ 0.175 and r.m.s. $\left(\log W_{r}(2796)-\log \hat{W}\right)=0.318$. We adopt $M_{B_{*}}-5 \log h=-19.8$ from Faber et al. (2007), which best characterizes the blue galaxy population at $z \sim 0.4$. The result of the likelihood analysis shows that including the scaling with $B$-band luminosity indeed reduces the intrinsic scatter by $\approx 12 \%$. At the same time, five outliers at $>3 \sigma_{c}$ from the mean relation (points in dotted circles) have now become apparent after accounting for the scaling with $B$-band luminosity.

Inspecting the imaging and spectral properties of the outliers, we note that the discrepant triangle and circle in the lower-center part of the graph are, respectively, an active galaxy showing broad emission lines and a red, evolved galaxy with a possible companion blended with a nearby bright star. The discrepant triangle in the upper-left is the galaxy-Mg II absorber pair with a large velocity separation of $\Delta v \approx-645 \mathrm{~km} \mathrm{~s}^{-1}$ in Figure 7 . The discrepant triangle and circle in the upper-right are galaxies with likely companions at close distances. Follow-up spectroscopy of these surrounding galaxies will confirm the nature of these close neighbors.

Excluding the five outliers, we obtain a revised best-fit power-law model of

$$
\begin{aligned}
\log \bar{W}_{r}^{\mathrm{p}}(2796)= & -(1.93 \pm 0.11) \log \rho \\
& -(0.27 \pm 0.02)\left(M_{B}-M_{B_{*}}\right) \\
& +(2.51 \pm 0.16),
\end{aligned}
$$

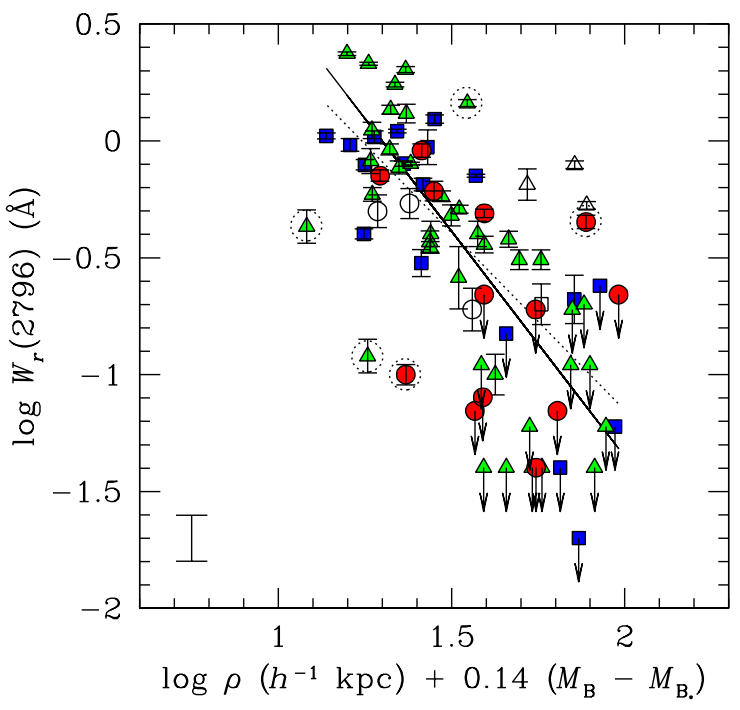

FIG. 9.- Comparison of $W_{r}(2796)$ versus $B$-band luminosity scaled impact parameter $\log \rho^{\prime}=\log \rho+a_{2}^{\prime}\left(M_{B}-M_{B_{*}}\right)$. The scaling coefficient $a_{2}^{\prime} \equiv a_{2} / a_{1}$ (Equation 6) is determined based on the likelihood analysis described in $\S$ 5.1 and is found to be $a_{2}^{\prime}=0.14 \pm 0.01$. Symbols are the same as in Figure 8. The dotted line is the best-fit power-law model for the entire sample of 71 galaxies, while the solid line is the best-fit power-law model, excluding five outliers (according to a 3- $\sigma$ clipping criterion; see $\$ 5.2$ ) marked in dotted circles. We note that the discrepant triangle and circle in the lower center part of the graph are, respectively, an active galaxy showing broad emission lines and a red, evolved galaxy with a possible companion blended with a nearby bright star. The discrepant triangle in the upper-left region is the galaxy$\mathrm{Mg}$ II absorber pair with a large velocity separation of $\Delta v \approx-645 \mathrm{~km} \mathrm{~s}^{-1}$ in Figure 7. The errorbar in the lower-left corner indicates the intrinsic scatter estimated from the likelihood analysis and defined in Equation (7).

with an intrinsic scatter of $\sigma_{c}=0.196$ and r.m.s.( $\log W_{r}-$ $\log \bar{W})=0.235$. We find that after accounting for an optimal scaling relation with $L_{B}$, the slope of the anti-correlation is steepened. We present the revised best-fit model as solid line in Figure 9. A generalized Kendall test demonstrates that the distribution of $W_{r}(2796)$ versus $\rho$ after accounting for galaxy luminosity deviates from a random distribution at more than 7- $\sigma$ level of significance. It indicates that the probability that $W_{r}(2796)$ is randomly distributed with respect to $\rho$ is $\ll 0.1 \%$.

\subsection{Dependence of Extended Gas on Galaxy Color and Redshift}

Here we examine whether including additional galaxy properties, such as $B_{A B}-R_{A B}$ color and redshift, helps to further strengthen this anti-correlation. Recall that our galaxy sample spans a range in $B_{A B}-R_{A B}$ color from $B_{A B}-R_{A B} \approx 0$ to $B_{A B}-R_{A B} \approx 1.5$, and a range in redshift from $z=0.1097$ to $z=0.4933$.

Including the intrinsic $B_{A B}-R_{A B}$ color of the galaxies as an additional independent variable in the power-law model and excluding the outliers in Figure 9, we find based on the likelihood analysis (Equations 7 through 9) that

$$
\begin{aligned}
\log \bar{W}_{r}^{\mathrm{p}}(2796)= & -(1.92 \pm 0.11) \log \rho \\
& -(0.27 \pm 0.02)\left(M_{B}-M_{B_{*}}\right) \\
& +(0.05 \pm 0.02)\left(B_{A B}-R_{A B}\right) \\
& +(2.46 \pm 0.15),
\end{aligned}
$$


with an intrinsic scatter of $\sigma_{c}=0.184$ and r.m.s.( $\log W_{r}-$ $\log \bar{W})=0.246$. The small scaling coefficient of the color term in Equation (12) indicates that the observed $W_{r}(2796)$ versus $\rho$ anti-correlation depends only weakly on galaxy intrinsic colors.

Next, we examine whether including galaxy redshift as an additional independent variable in the power-law model improves the strength of the anti-correlation. Excluding the outliers, we obtain a best-fit model of

$$
\begin{aligned}
\log \bar{W}_{r}(2796)= & -(1.90 \pm 0.11) \log \rho \\
& -(0.25 \pm 0.03)\left(M_{B}-M_{B_{*}}\right) \\
& +(0.73 \pm 0.50) \log \frac{(1+z)}{\left(1+z_{0}\right)} \\
& +(2.47 \pm 0.15),
\end{aligned}
$$

where $z_{0}=0.25$ which is the median redshift of the 'isolated' galaxy sample. The best-fit relation has an intrinsic scatter of $\sigma_{c}=0.184$ and r.m.s. $\left(\log W_{r}-\log \bar{W}\right)=0.245$. The uncertain scaling coefficient of the redshift term in Equation (13) indicates that there is little evolution in the extended $\mathrm{Mg}$ II halos around galaxies between $z \approx 0.1$ and $z \approx 0.5$.

\section{DISCUSSION}

We have established a spectroscopic sample of 94 galaxies at a median redshift of $\langle z\rangle=0.2357$ in fields around 70 distant background QSOs ( $\left.z_{\mathrm{QSO}}>0.6\right)$. Our follow-up MagE spectra of the QSOs allow us to examine the extent of cold gas around 88 of these galaxies based on the presence/absence of coincident $\mathrm{Mg}$ II absorption features.

In the sample of 88 spectroscopically confirmed galaxies, 17 are found in a 'group' environment with two or more close neighbors at $\rho<\hat{R}_{\text {gas }}$ (Equation 1) and velocity separation $\Delta v<300 \mathrm{kms}^{-1}$. Excluding one galaxy that occurs in the vicinity of the background QSO, we identify seven galaxy 'groups' along the lines of sight of the background QSOs in our sample. All seven galaxy 'groups' have coincident Mg II absorbers. Because the association between absorbers and individual galaxies becomes uncertain in a group environment, we have separated these group galaxies from the remaining 'isolated' ones. Figure 12 shows that while galaxies from a 'group' environment appear to occupy a similar range in the $W_{r}(2796)$ versus $\rho$ parameter space with 'isolated' galaxies, no strong inverse correlation is seen in the gaseous profiles of 'group' galaxies. Because interactions between group members are expected to alter the properties of gaseous halos, such as ram pressure and tidal stripping that could re-distribute cold gas to larger radii (e.g. Gunn \& Gott 1972; Balogh et al. 2000; Verdes-Montenegro et al. 2001), the 'group'-galaxy subsample also presents a unique opportunity to study gas kinematics in overdense galaxy environments beyond the local universe (c.f. Verdes-Montenegro et al. 2001).

The remaining 71 'isolated' galaxies are located at $\rho \lesssim$ $120 h^{-1} \mathrm{kpc}$ from the line of sight of a background QSO. We identify 47 coincident Mg II absorbers in the QSO spectra with absorber strengths varying from $W_{r}(2796)=0.1 \AA$ to $W_{r}(2796)=2.34 \AA$, and measure a sensitive upper limit of the $\mathrm{Mg}$ II absorber strength for the remaining 24 galaxies. In the absence of a complete spectroscopic survey of galaxies around the 71 'isolated' galaxies, it is likely that some fraction of these galaxies also occur in a group environment. However, the strong $W_{r}(2796)$ versus $\rho$ anti-correlation after accounting for the luminosity scaling relation seen for the 'isolated' galaxies in Figures $9 \& 12$ indicates that with the exception of a few outliers the majority of the 'isolated' galaxies are indeed different from those 'group' galaxies. We therefore argue that the majority of the 'isolated' galaxies are in a more quiescent environment than the 'group' ones in our sample.

The results of our likelihood analysis demonstrates that the $\mathrm{Mg}$ II absorber strength $W_{r}(2796)$ scales inversely with galaxy impact parameter. In addition, the $W_{r}(2796)$ vs. $\rho$ anticorrelation is still stronger after accounting for the scaling relation with galaxy $B$-band luminosity, indicating that more luminous galaxies are surrounded by more extended $\mathrm{Mg}$ II halos. However, including intrinsic $B_{A B}-R_{A B}$ color does not improve the observed $W_{r}(2796)$ vs. $\rho$ anti-correlation, indicating a lack of physical connection between the origin of extended $\mathrm{Mg}$ II halos and recent star formation history of the galaxies (c.f. Zibetti et al. 2007). Finally, the $W_{r}(2796)$ vs. $\rho$ anticorrelation appears to depend only weakly on galaxy redshift, indicating little evolution of the extended $\mathrm{Mg}$ II halos between $z \approx 0.5$ and $z \approx 0.1$. Here we focus on the 'isolated' galaxy sample and discuss the covering fraction and spatial profile of extended Mg II absorbing gas based on the observations and analysis presented in $\S 5$. We also discuss implications for the cool baryon content of galactic halos and compare our results with previous studies.

\subsection{Incidence and Covering Fraction of Mg II Absorbers}

The analysis presented in $\S 5.2$ shows that the strengths of $\mathrm{Mg}$ II absorbers depend on the projected distances and intrinsic luminosities of the absorbing galaxies. In addition to the best-fit scaling relation, we also note that with the exception of two outliers all 33 galaxies at luminosity-scaled impact parameter $\rho^{\prime} \equiv \rho \times\left(L_{B} / L_{B_{*}}\right)^{-0.35}<30 h^{-1}$ (Equation 11) have a coincident Mg II absorber of $W_{r}(2796)>0.3 \AA$. The observed high incidence of strong $\mathrm{Mg}$ II absorbers around galaxies with a broad range of intrinsic colors strongly supports the notion that extended $\mathrm{Mg}$ II halos are a common and generic feature of galaxies of all types, from evolved early-type galaxies to late-type star-forming systems.

While extended Mg II absorbing gas reaches out to larger projected distances, our sample shows that both the absorption strength and gas covering fraction declines toward larger radii. Here we examine how the gas covering fraction $\kappa$ varies with $\rho$ and $W_{r}(2796)$.

We perform a maximum-likelihood analysis to determine $\kappa$. The probability that a galaxy gives rise to an absorption system of some absorption equivalent width threshold is written as

$$
P(\kappa)=\kappa\left(\rho, W_{0}\right) B\left[r_{1}\left(L_{B}\right), r_{2}\left(L_{B}\right) ; \rho\right],
$$

where $\kappa$ is the fraction of galaxies that give rise to $\mathrm{Mg} \mathrm{II} \mathrm{ab-}$ sorption, and $B$ is a boxcar function that defines the impact parameter interval; $B=1$ if $r_{1}\left(L_{B}\right) \leq \rho<r_{2}\left(L_{B}\right)$ and $B=0$ otherwise. Equation (14) takes into account the scaling relation between the gaseous extent of galaxies and galaxy $B$-band luminosity. The likelihood of detecting an ensemble of galaxies, $n$ of which give rise to $\mathrm{Mg}$ II absorption systems and $m$ of which do not, is given by

$$
\begin{aligned}
\mathcal{L}(\kappa)= & \prod_{i=1}^{n} \kappa\left(\rho_{i}, W_{i}\right) B\left[r_{1}\left(L_{B_{i}}\right), r_{2}\left(L_{B_{i}}\right) ; \rho_{i}\right] \\
& \times \prod_{j=1}^{m}\left\{1-\kappa\left(\rho_{j}, W_{j}\right) B\left[r_{1}\left(L_{B_{j}}\right), r_{2}\left(L_{B_{j}}\right) ; \rho_{j}\right]\right\}
\end{aligned}
$$




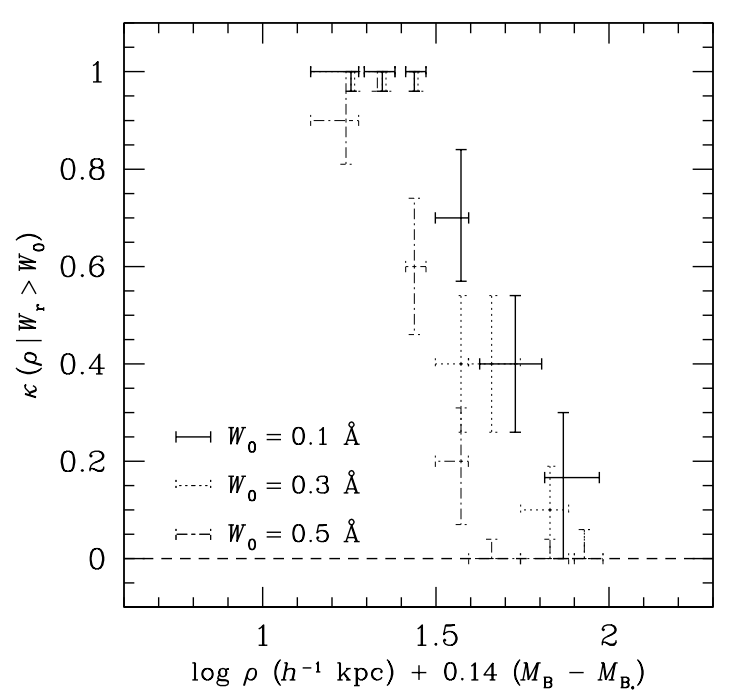

FIG. 10.- Incidence and covering fraction of $\mathrm{Mg} \mathrm{II}$ absorbers versus galaxy impact parameter accounting for the galaxy $B$-band luminosity scaling relation (Equation 11). Solid points indicate absorbers of $W_{r}(2796) \geq 0.1 \AA$ dotted points indicate absorbers of $W_{r}(2796) \geq 0.3 \AA$; and dot-dashed points indicate absorbers of $W_{r}(2796) \geq 0.5 \AA$. The impact parameter intervals are chosen to contain ten galaxy-absorber pairs per bin, except for the last bin that includes only the remaining seven pairs at largest separations. The errorbars represent the $68 \%$ confidence interval.

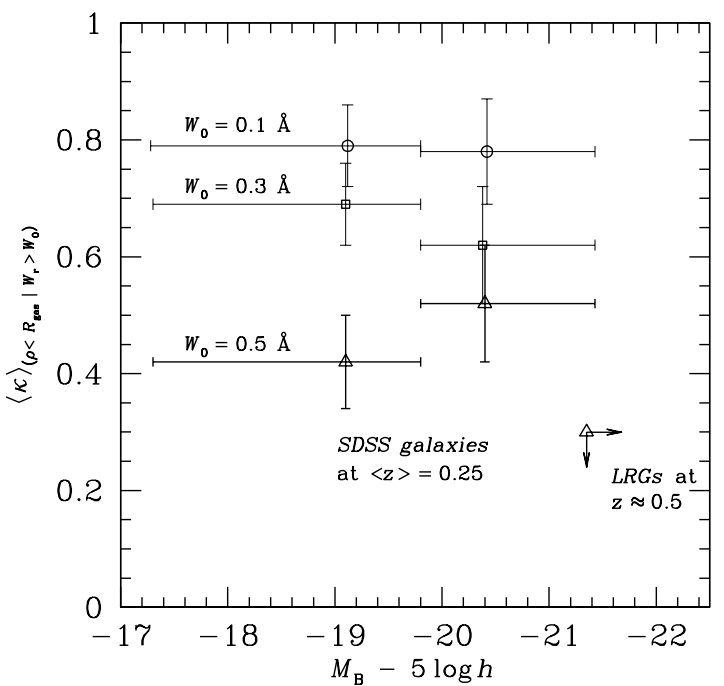

FIG. 11.- Mean covering fraction of Mg II absorbers within $R_{\text {gas }}$ of galaxies in different luminosity intervals. Circles represent a Mg II absorption threshold of $W_{0}=0.1 \AA$; squares represent $W_{0}=0.3 \AA$; and triangles represent $W_{0}=0.5 \AA$. The data points are located at the median absolute $B$-band magnitudes of the galaxies with horrizontal errorbars representing the luminosity intervals of the subsamples. Vertical errorbars represent the $68 \%$ confidence interval. For comparison, we have also included the limit of Gauthier et al. (2010) for Mg II absorbers with $W_{r}(2796) \geq 0.5 \AA$ around LRGs at $z \approx 0.5$.

We note that the LRGs are luminous with $M_{B}-5 \log h<$ -21.35 and are understood to reside in massive halos of $M_{h} \gtrsim 10^{13} h^{-1} \mathbf{M}_{\odot}$, whereas the galaxies in our sample are fainter, with a median rest-frame $B$-band magnitude of $\left\langle M_{B}-\right.$ $5 \log h\rangle=-19.6$, and presumably reside in lower-mass halos of $M_{h} \sim 10^{12.3} h^{-1} \mathrm{M}_{\odot}$ at $z \approx 0.25$. To examine how the covering fraction of $\mathrm{Mg}$ II absorbing gas varies with galaxy luminosity, we first divide our galaxy sample into luminous $\left(L_{B}>L_{B_{*}}\right)$ and faint $\left(L_{B} \leq L_{B_{*}}\right)$ subsamples and then determine the mean gas covering fraction $\left\langle\kappa_{W_{0}}\right\rangle$ over the entire gaseous halo defined by $R_{\text {gas }}$ (see $\S 6.2$ ) at a given $W_{r}(2796)$ threshold, $W_{0}$.

We consider three different threshold values, $W_{0}=0.1 \AA$, $W_{0}=0.3 \AA$, and $W_{0}=0.5 \AA$. The results are presented in Figure 11, together with the measurement of Gauthier et al. (2010) for $W_{0}=0.5 \AA$ around LRGs. The decreasing mean covering fraction over a fixed halo radius with increasing absorber strength is consistent with the expectation from a decreasing cloud density profile toward large radii (see $\S 6.2$ ). In addition, we find little dependence between the mean gas covering fraction and galaxy luminosity within our sample, but a significant reduction in gas covering fraction around LRGs. The declining gas covering fraction of strong Mg II absorbers with increasing galaxy luminosity (halo mass) is qualitatively consistent with the expectation from the observed clustering properties of Mg II absorbers (Tinker \& Chen 2008, 2009) and the theoretical expectation of diminishing cool gas fraction in massive halos (Kereš et al. 2005, 2009; Dekel \& Birnboim 2006).

\subsection{Density Profile of Extended Mg II Absorbing Gas} $\rho^{\prime}<30 h^{-1} \mathrm{kpc}$. 
The best-fit power-law model presented in Equation (11) and Figure 9 indicates that the observed absorber strength $W_{r}(2796)$ scales inversely with increasing projected distance $\rho$ with a steep slope of $\Delta \log W_{r}(2796) / \Delta \log \rho=-1.9$. While the power-law model appears to describe the observations well, a physical interpretation of this steep anti-correlation is not straightforward. Using a small sample of 13 galaxyMg II absorber pairs and 10 galaxies at $\rho<100 h^{-1} \mathrm{kpc}$ that do not give rise to $\mathrm{Mg}$ II absorption to a sensitive upper limit $\left(W_{r}(2796) \lesssim 0.02 \AA\right)$, Chen \& Tinker (2008) showed that the anti-correlation between $W_{r}(2796)$ and $\rho$, after accounting for $B$-band luminosity distribution, is well described by either an isothermal density profile or a Navarro-Frenk-White (NFW; Navarro et al. 1996) profile of the absorbing clumps with a finite extent $R_{\text {gas }}$. An isothermal density profile is motivated by the observed rotation curves of nearby galaxies, while an NFW profile is found to represent the density profiles of dark matter halos in high-resolution numerical simulations. Using the new sample of galaxy-Mg II absorber pairs that is three times of the Chen \& Tinker (2008) sample, we examine whether the anti-correlation displayed in Figure 9 is better described by either an isothermal density profile or an NFW profile.

For an isothermal profile of gaseous clumps within a finite extent $R_{\text {gas }}$, the $W_{r}(2796)$ versus $\rho$ relation is characterized following Chen \& Tinker (2008) as

$$
\bar{W}_{r}^{\text {iso }}(2796)=\frac{W_{0}}{\sqrt{\rho^{2} / a_{h}^{2}+1}} \tan ^{-1} \sqrt{\frac{R_{\mathrm{gas}}^{2}-\rho^{2}}{\rho^{2}+a_{h}^{2}}}
$$

at $\rho \leq R_{\text {gas }}$ and $W_{r}(2796)=0$ otherwise. The core radius $a_{h}$ is defined to be $a_{h}=0.2 R_{\text {gas }}$ and does not affect the expected $W_{r}(2796)$ at large $\rho$. The extent of $\mathrm{Mg}$ II absorbing gas scales with the luminosity of the absorbing galaxy according to

$$
\frac{R_{\text {gas }}}{R_{\text {gas } *}}=\left(\frac{L_{B}}{L_{B_{*}}}\right)^{\beta}
$$

Following the expectations of an isothermal model in Equations (16) and (17), we perform the likelihood analysis described in Equations (7) through (9) to find the best-fit values of $W_{0}, R_{\text {gas }_{*}}$, and $\beta$. Excluding the outliers, the results of the likelihood analysis show that the observations are best described by $\log W_{0}^{\text {iso }}=1.24 \pm 0.03$,

$$
\beta^{\text {iso }}=0.35_{-0.04}^{+0.01}
$$

and

$$
\log R_{\text {gas }_{*}}^{\text {iso }}=1.87 \pm 0.01 .
$$

The errors indicate the $95 \%$ confidence intervals. The best-fit isothermal profile is also characterized by an intrinsic scatter of $\sigma_{c}=0.104$ and an r.m.s. residual between the observed and model Mg II absorber strengths of r.m.s. $\left(\log W_{r}-\log \bar{W}\right)=$ 0.233 .

For an NFW profile, the absorber strength is expected to vary with $\rho$ according to

$$
\bar{W}_{r}^{\mathrm{NFW}}(2796)=\int_{0}^{\sqrt{R_{\text {gas }}^{2}-\rho^{2}}} \frac{W_{0} r_{s}^{3}}{\left(r_{s}+\sqrt{l^{2}+\rho^{2}}\right)^{2} \sqrt{l^{2}+\rho^{2}}} d l
$$

where $r_{s}$ is the scale radius. We adopt $r_{s} \equiv R_{200} / 15$ that gives a halo concentration index of 15 (e.g. Dolag et al. 2004). We perform the likelihood analysis described in Equations (7) through (9) and find $\log W_{0}^{\mathrm{NFW}}=-0.56 \pm 0.05$,

$$
\beta^{\mathrm{NFW}}=0.35 \pm 0.03 \text {, }
$$

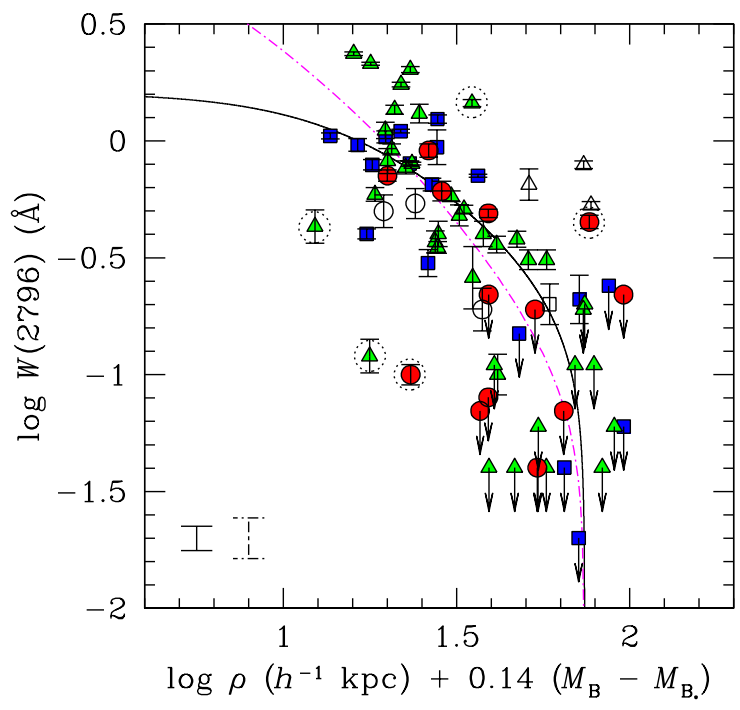

FIG. 12.- Comparison of $W_{r}(2796)$ versus impact parameter accounting for scaling by $B$-band luminosity and redshift. Symbols are the same as in Figure 8 . The solid curve is the best-fit isothermal model and the dash-dotted curve is the best-fit NFW model, excluding five outliers (according to a 3- $\sigma$ clipping criterion; see $\S 5.2$ ) marked in dotted circles. The errorbars in the lower-left corner indicate the intrinsic scatters of the adopted model profiles estimated based on the likelihood analysis. We note that the residuals between observations and the best-fit model profile do not correlate with galaxy $B$-band luminosity, indicating that $W_{0} / R_{\text {gas }}$ in Equation (16) does not depend on galaxy luminosity.

and

$$
\log R_{\mathrm{gas}_{*}}^{\mathrm{NFW}}=1.89_{-0.12}^{+0.05} \text {. }
$$

The errors indicate the $95 \%$ confidence intervals. The bestfit NFW profile has associated intrinsic scatter of $\sigma_{c}=0.175$ and r.m.s. residual between the observed and model $\mathrm{Mg}$ II absorber strengths of r.m.s. $\left(\log W_{r}-\log \bar{W}\right)=0.253$.

Figure 12 displays the best-fit models in comparison to observations. While the isothermal model provides a somewhat better characterization of the observations in terms of a minimum derived intrinsic scatter and r.m.s. residual, both models result in a consistent best-fit scaling relation of

$$
\frac{R_{\mathrm{gas}}}{R_{\mathrm{gas}_{*}}}=\left(\frac{L_{B}}{L_{B_{*}}}\right)^{0.35},
$$

where the characteristic gaseous radius of an $L_{*}$ galaxy is found to be

$$
R_{\mathrm{gas}_{*}} \approx 75 h^{-1} \mathrm{kpc}
$$

at $z \sim 0.25$.

It is clear that the scatter between the observations and the best-fit isothermal model after accounting for the scaling relation with galaxy luminosity remains large in Figure 12. A natural expectation of the isothermal model in Equation (16) is that $W_{0}$ depends on galaxy properties, particularly on $L_{B}$ or mass, because more luminous (and therefore more massive) galaxies possess more extended gaseous halos and would exhibit stronger $W_{0}$ (e.g. Tinker \& Chen 2010). However, we find no correlation between the residuals of $\left(\log W_{r}-\log \bar{W}\right)$ in Figure 12 and absolute $B$-band magnitude of the galaxies, indicating that $W_{0}$ does not depend strongly on galaxy luminosity/mass. The lack of correlation suggests that the mean 
absorber strength per halo does not vary with halo mass and that the cool gas fraction declines with increasing halo mass in the mass regime probed by our sample.

The best-fit scaling power in Equation (23) confirms the earlier result of Chen \& Tinker (2008). The best-fit characteristic gaseous radius shown in Equation (24) is smaller than the earlier estimate of $91_{-8}^{+3} h^{-1} \mathrm{kpc}$ from a smaller galaxy sample, but they are consistent to within 2- $\sigma$ error uncertainties. The new galaxy sample presented in this paper, which is three times larger than the initial sample employed by Chen \& Tinker, has revealed a substantial scatter, particularly at large radii. The large uncertainty in $R_{\text {gas }_{*}}$ can be understood as due to the inherent degeneracy between the spatial extent $R_{\text {gas }}$ and diminishing covering fraction $\kappa(\rho)$ of $\mathrm{Mg}$ II absorbing gas at large radii. A larger $R_{\mathrm{gas}_{*}}$ can be accommodated with a smaller gas covering fraction. For example, within $R_{\mathrm{gas}_{*}}=75 h^{-1} \mathrm{kpc}, 39$ of the 59 galaxies have an associated Mg II absorber of $W_{r}(2796) \geq 0.3 \AA$. This yields a gas covering fraction of $\kappa_{0.3} \approx 66 \%$ at $\rho^{\prime}<75 h^{-1} \mathrm{kpc}$. Within $R_{\text {gas }_{*}}=91 h^{-1} \mathrm{kpc}$, we find $\kappa_{0.3} \approx 61 \%$ at $\rho^{\prime}<91 h^{-1} \mathrm{kpc}$ after excluding outliers.

In Chen \& Tinker (2008), the best-fit $R_{\mathrm{gas}_{*}}=91 h^{-1} \mathrm{kpc}$ was close to the maximum impact separation of $r_{0}=100 h^{-1} \mathrm{kpc}$ in the galaxy-QSO pair sample. The selection criterion was imposed by these authors to minimize the incidence of correlated galaxies through large-scale clustering (see their Section 2 for discussion). Chen \& Tinker examined the possible bias in the best-fit $R_{\mathrm{gas}_{*}}$ due to the imposed $r_{0}$ selection, and found that the best-fit $R_{\mathrm{gas}_{*}}$ was insensitive to the choice of $r_{0}$. Namely, they obtained a consistent estimate of $R_{\mathrm{gas}_{*}}$ for a smaller $r_{0}$. However, choosing a smaller $r_{0}$ reduced the already small sample further and the parameters became poorly constrained.

In the present analysis, we have included galaxy-QSO pairs with impact separation as large as $\rho \approx 120 h^{-1} \mathrm{kpc}$ (Figure 3). The best-fit gaseous radius of $R_{\mathrm{gas}_{*}}=75 h^{-1} \mathrm{kpc}$ is significantly below the the maximum separation in the pair sample, although the majority of the data (galaxies with intrinsic colors consistent with present-day disks and irregular's/starbursts) lie at $\rho<75 h^{-1} \mathrm{kpc}$. To examine whether the best-fit $R_{\mathrm{gas}_{*}}$ is sensitive to the range of impact parameter covered by the pair sample, we repeat the likelihood analysis adopting only pairs with $\rho<75 h^{-1} \mathrm{kpc}$. We find that the best-fit results remain the same.

In summary, we find based on a sample of 71 'isolated' galaxies that the extent of cool gas as probed by the $\mathrm{Mg}$ II absorption features scales with galaxy $B$-band luminosity according to $R_{\text {gas }} \propto L_{B}^{0.35}$, consistent with earlier results obtained using smaller samples. As discussed in Chen \& Tinker (2008), the scaling relation, when combined with a fiducial mass-to-light ratio determined from galaxy halo occupation studies of wide-field galaxy survey data, indicates that the gaseous radius is a constant fraction of the halo radius over the mass scale of $M_{h}=10^{10.6}-10^{13} h^{-1} \mathrm{M}_{\odot}$ sampled in our observations ${ }^{9}$. In addition, we find that the residuals of observed

\footnotetext{
9 The halo occupation analysis of $2 \mathrm{dFGRS}$ galaxies from Tinker et al. (2007) showed that the relationship between $M_{h}$ and luminosity for galaxies of $L_{b_{J}} \lesssim L_{*}$ at $z \sim 0.1$ is $M_{h}=10^{12.5}\left(L_{B} / L_{B_{*}}\right)^{1.3} h^{-1} \mathrm{M}_{\odot}$. This monotonic relationship is appropriate for galaxies that reside at the centers of their dark matter halos and are the brightest galaxy in the halo. A similar scaling relation was obtained, $M_{h}=10^{11.9}\left(L_{B} / L_{B_{*}}\right)^{1.3} h^{-1} \mathrm{M}_{\odot}$, for DEEP2 galaxies at $z \sim 1$ by Zheng et al. (2007). Interpolating in $\log (1+z)$, we find that galaxies of $M_{B_{*}}-5 \log h=-19.8$ on average reside in halos of $10^{12.4} h^{-1} \mathrm{M}_{\odot}$ at $z \sim 0.25$.
}

and best-fit $W_{r}(2796)$ do not correlate with galaxy absolute $B$ band magnitude, implying a declined cold gas fraction with increasing halos mass in the mass regime probed by our sample.

\subsection{The Origin of Extended Cool Gas and Implications for the Baryon Content of Galactic Halos}

The presence of a finite extent $R_{\text {gas }}$ of $\mathrm{Mg}$ II absorbing gas is similar to what is found for extended C IV (Chen et al. 2001) and also for OVI absorbing gas recently published by Chen \& Mulchaey (2009) around galaxies. The origin of such a finite boundary for metal-line absorbers can be interpreted as due to a halo fountain phenomenon (c.f. Bregman 1980), according to which the gaseous radius is driven by the finite distance the outflowing material can travel from an early episode of starburst. We find this scenario unlikely because of the broad range of intrinsic colors covered by our galaxy sample, but a detailed stellar population synthesis to investigate the star formation history will shed more light on this issue.

Alternatively, the finite boundary of absorbing clouds probed by these metal-line absorbers can be understood as a critical radius below which cool clouds can form and survive in an otherwise hot medium. This two-phase model to interpret QSO absorption line systems was formulated in Mo \& Miralda-Escudé (1996) and later re-visited by Maller \& Bullock (2004). This is also shown in recent high-resolution numerical simulations of Milky Way type halos, in which accreted cold streams are disrupted by shocks but the remaining gas overdensities serve as the 'seeds' necessary to form cool gaseous clouds through thermal instabilities within $\approx 1 / 3$ of the halo radius (e.g. Kereš \& Hernquist 2009).

The formation and presence of such cool clouds in a hot halo have several important implications for the studies of galaxy formation and evolution. For example, the confining hot medium around the cool clouds is expected to have a low density and long cooling time, reducing the "overcooling problem" in standard galaxy formation models (e.g. Maller \& Bullock 2004; Kaufmann et al. 2009). In addition, these clouds may provide the fuels necessary to support continuous star formation near the center of galactic halos (e.g. Binney et al. 2000). Furthermore, the cool clouds together with the confining hot medium offer a reservoir for missing baryons in individual galactic halos (e.g. McGaugh et al. 2010). A nominal candidate for these cool clouds in our Milky Way Halo is the high velocity clouds (HVCs) of neutral hydrogen column density $N(\mathrm{HI})>10^{18} \mathrm{~cm}^{-2}$ seen in all-sky $21 \mathrm{~cm}$ observations. However, unknown distances of these HVCs prohibit an accurate measurement of their total gas mass (e.g. Putman 2006).

High-resolution spectra of strong Mg II absorbers have revealed the multi-component nature of the absorbing gas, with $W_{r}(2796)$ roughly proportional to the number of components in the system (e.g. Petitjean \& Bergeron 1990; Prochter et al. 2006). These discrete Mg II absorbing components are natural candidates of the condensed cool clouds within $\sim 75 \mathrm{~h}^{-1}$ $\mathrm{kpc}$ radius of known galaxies. Interpreting the intrinsic scatter $\sigma_{c}$ as due to Poisson noise in the number of cool clumps intercepted along a line of sight allows us to estimate the number of absorption clumps per galactic halo per sightline, $n^{\text {clump}}$. In this simple model, each absorber is characterized as $W_{r}=n^{\text {clump }} \times k$, where $k$ is the mean absorption equivalent width per absorbing component. Following Poisson counting statistics, the intrinsic scatter $\sigma_{c} \equiv \sigma_{c, W_{r}} /\left(W_{r} \ln 10\right)$ is related 
to $n^{\text {clump }}$ according to

$$
\sigma_{c}=\frac{1}{\ln 10} \frac{\sqrt{n^{\text {clump }}+1}}{n^{\text {clump }}}
$$

For the isothermal model, we have determined a best-fit intrinsic scatter of $\sigma_{c}=0.104$, which leads to a mean number of absorbing clumps of

$$
n^{\text {clump }} \sim 18
$$

and a mean absorption equivalent width per clump of $k=0.04$ $\AA$ at a median projected distance of $\langle\rho\rangle=26 h^{-1} \mathrm{kpc}$. The corresponding $\mathrm{Mg}$ II absorbing column density per clump is $N(\mathrm{Mg}$ II $) \approx 10^{12} \mathrm{~cm}^{-2}$. We note that if other factors contribute to the observed $\sigma_{c}$, then the corresponding Poisson noise is smaller and the inferred $n^{\text {clump }}$ will be bigger.

To estimate the total baryonic mass contained in these cool clouds traced by $\mathrm{Mg}$ II absorbers, we first assume a mean clump size of $1 \mathrm{kpc}$ (consistent with the size of $\mathrm{HI}$ clouds seen around M31; Westmeier et al. 2008), a mean metallicity of 1/10 solar (consistent with what is seen in the HVC Complex C; see Thom et al. 2008 for a list of references), and a mean ionization fraction of $f_{\mathrm{Mg}^{+}}=0.1$ for photo-ionized clouds (see Figure 6 of Chen \& Tinker 2008). We derive a mean gas density of $n_{\mathrm{H}} \approx 10^{-3} \mathrm{~cm}^{-3}$. Assuming a spherical shape for the clumps leads to a mean clump mass of $M_{b}^{\text {clump }}=1.7 \times 10^{4} \mathrm{M}_{\odot}$. Next, we adopt an isothermal density distribution of the clumps (Equation 16) and derive a total number of clumps $N^{\text {clump }}=2 \times 10^{5} h^{-2}$ within $R_{\text {gas }}=75 h^{-1}$ $\mathrm{kpc}$. The total baryonic mass in these cool clumps is found to be $M_{b} \sim 3 \times 10^{9} h^{-2} \mathrm{M}_{\odot}$, comparable to the total cold gas content seen in the Milky Way disk (e.g. Flynn et al. 2006). The inferred total mass would be still larger if $n^{\text {clump }}$ increases as the Poisson noise decreases.

If we further assume that it takes a free-fall time for the clumps to reach the center of the halo, we infer a cool gas accretion rate of $\dot{M}_{\text {gas }} \sim 3 \mathrm{M}_{\odot} \mathrm{yr}^{-1}$. The inferred gas accretion rate is interestingly close to the star formation rate seen in the Milky Way disk (e.g. Robitaille \& Whitney 2010). However, we note two issues in this simple picture.

The first issue concerns the lifetime of these cool clumps relative to the time it takes for them to reach the inner few $\mathrm{kpc}$ of the host halo. Previous studies have shown that clouds of mass $M_{c} \lesssim 10^{5} \mathrm{M}_{\odot}$ cannot survive thermal conduction and ram pressure as they move through a low-density, hot medium (see e.g. Mo \& Miralda-Escudé 1996; Maller \& Bullock 2004; Heitsch \& Putman 2009). To alleviate this problem, the clumps will need to have lower metallicities and/or bigger sizes than what we have assumed. For example, if we assume a mean clump size of $2 \mathrm{kpc}$ and a mean metallicity of 0.05 solar, then we derive a mean clump mass of $M_{b}^{\text {clump }}=1.3 \times 10^{5} \mathrm{M}_{\odot}$. The total number of clumps would be $N^{\text {clump }}=5 \times 10^{4} h^{-2}$ and the total cool gas mass would be $M_{b} \sim 6.5 \times 10^{9} h^{-2} \mathrm{M}_{\odot}$ at $r \leq 75 h^{-1} \mathrm{kpc}$.

Second, we note that the expected H I column density of these Mg II absorbing clumps is $N(\mathrm{HI}) \sim 10^{16} \mathrm{~cm}^{-2}$, below the $N(\mathrm{HI})$ threshold provided by $21 \mathrm{~cm}$ observations. The $\mathrm{HI}$ content is therefore too low for these clumps to be the distant counterparts of the local HVCs. Nevertheless, the lack of strong $\left(W_{r}(2796) \geq 0.5 \AA\right) \mathrm{Mg}$ II absorbers beyond $30 \mathrm{~h}^{-1}$ kpc (Figure 10) which have have associated H I of $N(\mathrm{HI})>$ $10^{18} \mathrm{~cm}^{-2}$ (Rao et al. 2006), constrains the distances of the Milky Way HVCs at $d \lesssim 50 \mathrm{kpc}$. Within this close distance range, the implied total gas mass in the HVCs is expected to be $M_{b}^{\mathrm{HVC}}<10^{9} \mathrm{M}_{\odot}($ Putman 2006).

The exercise presented above shows that the cool clouds that give rise to $\mathrm{Mg}$ II absorbers and the implied presence of a hot confining medium can provide a substantial reservoir for missing baryons in individual galactic halos (see also Kaufmann et al. 2009). The presence of extended Mg II absorbing halos around galaxies of a broad range of intrinsic colors argues against an outflow origin of these absorbers. Under an infall scenario, we show that these cool clouds provide sufficient fuels to support continuous star formation in their host galaxies.

\subsection{Comparison with Other Studies}

The large covering fraction of Mg II absorbing gas ( $\$ 6.1$ ) and the scaling relation between gaseous extent and galaxy $B$-band luminosity ( $\$ 6.2$ ) represent the empirical evidence supporting the halo occupation model developed in Tinker \& Chen (2008, 2009; see also Gauthier et al. 2009). Here we compare the results of our studies with recent surveys by other groups.

First, we have noted the discrepancy between the high gas covering fraction around $\sim L_{*}$ galaxies and the low incidence of $\mathrm{Mg}$ II absorbers around luminous red galaxies studied in Gauthier et al. (2010). We have interpreted the contrast as due to the different halo mass scales probed by the two galaxy samples. Cosmological simulations have shown that the growth of galaxies progresses primarily through stable accretion of intergalactic gas (e.g. Kereš et al. 2005; 2009). In low-mass halos $\left(M_{h}<M_{h}^{\text {crit }}=10^{11.5} h^{-1} \mathrm{M}_{\odot}\right)$, accretion proceeds to the center of the halo through dense cold streams (Dekel \& Birnboim 2006; Kereš et al. 2005; 2009). As galaxies grow larger in mass $\left(M_{h}>M_{h}^{\text {crit }}=10^{11.5} h^{-1} \mathrm{M}_{\odot}\right)$, both numerical simulations and analytic models show that a progressively larger fraction of the accreted gas is shock heated to the virial temperature. Some fraction of the shock-heated gas may be able to cool in the inner region where gas density is high. The majority of the galaxies presented in this paper are on the transitional mass scale, $M_{h}=10^{11-12.5} h^{-1} \mathrm{M}_{\odot}$. In contrast, the luminous red galaxies studied by Gauthier et al. (2010) are in a higher mass regime of $M_{h}>10^{13} h^{-1} \mathrm{M}_{\odot}$, where a diminishing amount of cold gas is expected because the cooling time is long. The differential gas covering fraction found in our galaxies and in those luminous red galaxies is therefore consistent with theoretical expectations.

Kacprzak et al. (2008) have also studied the extent and covering fraction of $\mathrm{Mg}$ II absorbing gas based on a sample of 37 galaxies at $z=0.3-1$ that are found in the vicinity of a known Mg II absorber. While they did not see a clear correlation between the extent of Mg II absorbing gas and galaxy luminosity, they found that for a scaling power of $\beta=0.18-0.58$ and a characteristic gaseous extent of $R_{*}=56-105 h^{-1} \mathrm{kpc}$ the covering fraction of $\mathrm{Mg}$ II absorbers with $W_{r}(2796) \geq 0.3 \AA$ is no more than $70 \%$. Our best-fit isothermal model indicates that the characteristic gaseous radius at $W_{r}(2796)=0.3 \AA$ is $R_{\text {gas }_{*}}\left(W_{r}=0.3\right)=47 h^{-1} \mathrm{kpc}$ (Figure 11), less than the gaseous radius considered by Kacprzak et al.. Excluding the outliers, we find a mean covering fraction of $\kappa_{0.3}\left[\rho^{\prime}<47 h^{-1} \mathrm{kpc}\right] \approx$ $76 \%$ for $\mathrm{Mg}$ II absorbers with $W_{r}(2796) \geq 0.3 \AA$. Including the outliers, we find a mean covering fraction of $\kappa_{0.3}\left[\rho^{\prime}<\right.$ $\left.47 h^{-1} \mathrm{kpc}\right] \approx 78 \%$ for $\mathrm{Mg}$ II absorbers with $W_{r}(2796) \geq 0.3$ $\AA$. The gas covering fraction found in our sample is somewhat higher than the parameter space explored by Kacprzak 
et al., but this can be explained by the larger gaseous radius considered by these authors.

In addition, Barton \& Cooke (2009) have recently carried out a search of coincident $\mathrm{Mg}$ II absorbers in the vicinity of 20 luminous galaxies $\left(M_{r}-5 \log h \leq-20.5\right)$ identified at $z \sim 0.1$ in the SDSS galaxy spectroscopic sample. The authors reported a low gas covering fraction of $\kappa_{0.3}\left[\rho<75 h^{-1} \mathrm{kpc}\right] \lesssim$ 0.4 and $\kappa_{0.3}\left[\rho<35 h^{-1} \mathrm{kpc}\right] \sim 0.25$ for $\mathrm{Mg}$ II absorbers of $W_{r}(2796) \geq 0.3 \AA$. These results are inconsistent with the high gas covering fraction found in our survey. The design of the Barton \& Cooke survey is very similar to ours presented here, with only small differences in the targeted redshift range $(z \sim 0.1)$ and luminosity scale $\left(>L_{*}\right)$. The discrepancy in the observed gas covering fraction therefore requires further perusal.

A fundamental difference in our respective measurements is that Barton \& Cooke excluded observed strong Mg II absorbers in the covering fraction calculation if the QSO spectrum did not have sufficient $S / N$ for identifying absorbers of $W_{r}(2796)=0.3 \AA$. Of the six galaxies at $\rho \leq 35 h^{-1} \mathrm{kpc}$, three have associated $\mathrm{Mg}$ II absorbers of $W_{r}(2796)=1.93 \pm 0.18$ $\AA, W_{r}(2796)=1.91 \pm 0.10 \AA$, and $W_{r}(2796)=0.71 \pm 0.15 \AA$, respectively, and three do not have corresonding $\mathrm{Mg}$ II absorbers to $3-\sigma$ limits of $W_{r}(2796)<0.3 \AA$. The authors excluded from the covering fraction calculation two strong absorbers, $W_{r}(2796)=1.93 \pm 0.18 \AA$ and $W_{r}(2796)=0.71 \pm 0.15$ $\AA$, and derived $\kappa_{0.3}\left[\rho<35 h^{-1} \mathrm{kpc}\right] \sim 0.25$ based on the remaining four galaxies.

We note that the goal of our study is to determine the incidence of $\mathrm{Mg}$ II absorbers at the location of a known galaxy. Absorbers that are observed to have $W_{r}(2796)=0.71 \pm 0.15$ $\AA$ and $W_{r}(2796)=1.93 \pm 0.18 \AA$ confirm the presence of such absorbing gas in large quantities, despite the fact that the QSO spectrum may not offer the sensitivities required for uncovering weaker absorbers of $W_{r}(2796) \sim 0.3 \AA$ at high confidence levels. Excluding these sightlines imposes a bias that would result in an underestimate of the gas covering fraction.

We have retrieved from the SDSS data archive the galaxy photometric and spectroscopic data in Barton \& Cooke (2009), and determined the rest-frame $B$-band absolute magnitude and $B_{A B}-R_{A B}$ color for each of the 20 galaxies according to the procedures described in $\S 4.1$. The rest-frame absolute $B$-band magnitudes of these galaxies span a range from $M_{B}-5 \log h=-19.6$ to $M_{B}-5 \log h=-20.7$ with a median of $\left\langle M_{B}-5 \log h\right\rangle=-20.3$. The rest-frame $B_{A B}-R_{A B}$ colors range from $B_{A B}-R_{A B} \approx 0.6$ to $B_{A B}-R_{A B} \approx 1.2$ with a median of $\left\langle B_{A B}-R_{A B}\right\rangle_{\text {med }} \approx 1$ 1.0. Figure 13 displays the observed $W_{r}(2796)$ versus $\rho$ relation for the Barton \& Cooke sample, superimposed on top of our own data. We have converted the 3- $\sigma$ upper limits published by these authors to 2- $\sigma$ upper limits in order to be consistent with our own measurements.

Figure 13 shows that with the exception of two outliers at $\rho^{\prime} \approx 25 h^{-1} \mathrm{kpc}$, the Barton \& Cooke sample is in general agreement with what is seen in our sample. Six of the 12 upper limits in the Barton \& Cooke sample do not have sufficient sensitivities for constraining the presence/absence of $\mathrm{Mg}$ II absorbing gas at the level of $W_{r}(2796) \sim 0.3$ $\AA$. In addition, the galaxy at $\log \rho^{\prime}=\log \rho+0.14\left(M_{B}-\right.$ $\left.M_{B_{*}}\right) \approx 1.6$ with a strong $\mathrm{Mg}$ II absorber of $W_{r}(2796)=1.24$ (SDSSJ144033.82+044830.9) was later found to have a companion galaxy at $\rho=18.6 h^{-1} \mathrm{kpc}$. The absorber would therefore be considered as in a 'group' environment. The galaxy at $\rho^{\prime} \approx 20 h^{-1} \mathrm{kpc}$ with a $2-\sigma$ upper limit of $W_{r}(2796)<0.13$

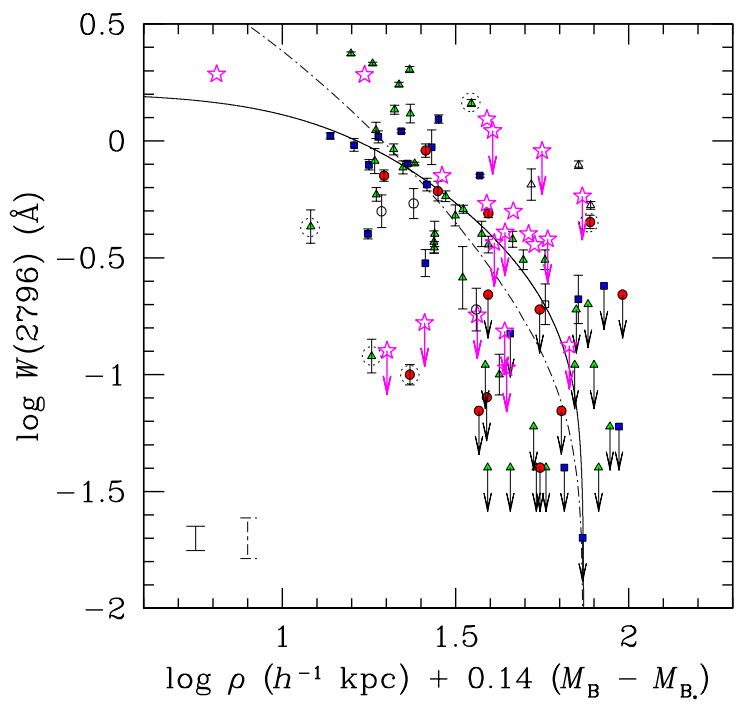

FIG. 13. - Comparison of the observed $W_{r}(2796)$ versus $\rho$ correlation for galaxies in our sample and those published in Barton \& Cooke (2009). We have applied the scaling relation of Equation (23) for both samples. Symbols and best-fit models for our sample are the same as in Figure 11. The galaxies of Barton \& Cooke are shown in star symbols. We note that the galaxy at $\log \rho^{\prime}=\log \rho+0.14\left(M_{B}-M_{B_{*}}\right) \approx 1.6$ with a strong $\mathrm{Mg}$ II absorber of $W_{r}(2796)=1.24($ SDSSJ144033.82+044830.9) in the Barton \& Cooke sample has been found to have a companion galaxy at $\rho=18.6 h^{-1} \mathrm{kpc}$. The absorber would therefore be considered as in a 'group' environment.

$\AA$ (SDSSJ151541.23+334739.4) has a luminous companion (SDSSJ151536.18+334743.6) at projected distance $<100 \mathrm{~h}^{-1}$ $\mathrm{kpc}$ and velocity separation $\Delta v \approx 27 \mathrm{~km} \mathrm{~s}^{-1}$ away. This pair also respresents a 'group' that we have treated separately in our analysis.

Considering only pairs between 'isolated' galaxies and QSOs for which sensitive limits for the corresponding Mg II absorption are available leads to a sample of 12 galaxies with sensitive constraints for the corresponding $\mathrm{Mg}$ II absorption features. We find three of the four galaxies at $\rho^{\prime}<35 h^{-1} \mathrm{kpc}$ have associated Mg II absorbers of $W_{r}(2796)>0.3 \AA$, yielding $\kappa_{0.3}\left[\rho^{\prime}<35 h^{-1} \mathrm{kpc}\right] \approx 0.75$. We find seven of the 12 galaxies at $\rho^{\prime}<75 h^{-1} \mathrm{kpc}$ have associated Mg II absorbers of $W_{r}(2796)>0.3 \AA$, yielding $\kappa_{0.3}\left[\rho^{\prime}<75 h^{-1} \mathrm{kpc}\right] \approx 0.58$. These values are consistent with our estimated gas covering fraction to within 1- $\sigma$ uncertainties. We therefore conclude that the observations presented in Barton \& Cooke (2009) are consistent with the high gas covering fraction seen in our sample.

Finally, Zibetti et al. (2007) studied the nature of Mg II absorbing galaxies at $z=0.37-1$ based on stacked images of QSOs with known foreground $\mathrm{Mg}$ II absorbers of $W_{r}(2796)>$ $0.8 \AA$. After subtracting the QSO point spread function in the stacked images, these authors obtained an angular averaged image of $\mathrm{Mg}$ II absorbing galaxies out to $100 \mathrm{kpc}$. They found that the luminosity-weighted mean colors of the extended emission is consistent with present-day intermediatetype galaxies. In addition, the authors found that weak absorbers of $W_{r}(2796)<1.1 \AA$ originate primarily from red passive galaxies while stronger absorbers display bluer colors consistent with star-forming galaxies. Based on the dif- 
ferential color distribution, Zibetti et al. argued that the origin of strong Mg II absorbers might be better explained by models of metal-enriched gas outflows from star-forming/bursting galaxies.

Our analysis in $\S 5.3$ shows that the observed Mg II absorber strength does not depend strongly on galaxy intrinsic color, which appears to contradict the observations of $\mathrm{Zi}$ betti et al.. To understand the discrepant results, we first recall based on the observed $W_{r}(2796)$ versus $\rho$ anti-correlation in Figure 8 that the mean impact parameter of the absorbing galaxies for weak absorbers is expected to be larger than that for stronger absorbers. In addition, Figure 8 also shows that for a sample of randomly selected QSO-galaxy pairs in our study, the fraction of red galaxies is found to be a factor of two larger at larger impact parameters $\rho>30 h^{-1} \mathrm{kpc}$ than at closer distances. This is not due to selection biases, because we did not preferentially select blue galaxies at smaller impact parameters. Instead, this can be understood by considering the fact that redder galaxies are on average more luminous and have a lower space density than bluer and on average fainter galaxies (e.g. Faber et al. 2007). The increased incidence of red galaxies at larger impact parameter, combined with the luminosity-weighted nature of the image stacking procedure, is expected to skew the stacked images of weaker absorbers toward redder colors. Whether or not this is sufficient to explain the observed color gradient between strong and weak Mg II absorbers in the Zibetti et al. sample requires a detailed simulation that takes into account such intrinsic weighting by the space density and luminosity of the galaxies. Because such analysis has not been performed, it is not clear whether the respective results are inconsistent with each other. Nevertheless, the conclusion in favor of strong $\mathrm{Mg}$ II absorbers being better explained by starburst outflows based on the observed color gradient should be viewed with caution.

\section{SUMMARY}

We are conducting a survey of $\mathrm{Mg}$ II absorbers in the spectra of background QSOs that are within close angular distances to a foreground galaxy at $z<0.5$, using the Magellan Echellette Spectrograph. The goal of this project is to establish a statistically significant sample $(N \gtrsim 500)$ of galaxies with sensitive constraints on their surrounding gaseous halos for measuring the clustering amplitude of $\mathrm{Mg}$ II absorbers at $\langle z\rangle=0.2$ and for a comprehensive study of how the properties of extended cool gas correlate with known stellar and ISM properties of the host galaxies.

Here we report the results of the first-year survey data. We have so far established a spectroscopic sample of 94 galaxies at a median redshift of $\langle z\rangle=0.2357$ in fields around 70 distant background QSOs $\left(z_{\mathrm{QSO}}>0.6\right)$. The galaxies span a broad range in the intrinsic $B_{A B}-R_{A B}$ color, from $B_{A B}-R_{A B} \approx$ 0 to $B_{A B}-R_{A B} \approx 1.5$, and a broad range in the rest-frame absolute $B$-band magnitude, from $M_{B}-5 \log h=-16.22$ to $M_{B}-5 \log h=-22.5$. Of the 94 galaxies, 17 are found with at least one neighbor at close projected distances $\rho<\hat{R}_{\text {gas }}$ (defined in Equation 1) and velocity separations $\Delta v<300$ $\mathrm{km} \mathrm{s}^{-1}$; one occurs at $z=0.0934$, outside of the redshift range allow by the spectral coverage of MagE for the $\mathrm{Mg}$ II absorber search; and five do not have sensitive limits for $W_{r}(2796)$ due to contamination by other absorption features. Excluding these galaxies leads to a sample of 71 'isolated' galaxies at $\rho \lesssim 120 h^{-1} \mathrm{kpc}$ from the line of sight of a background QSO. Of the 71 galaxies, we find that 47 have corresponding $\mathrm{Mg}$ II absorbers in the spectra of background QSOs with absorber strengths varying from $W_{r}(2796)=0.1 \AA$ to $W_{r}(2796)=2.34$ $\AA$, and 24 do not give rise to $\mathrm{Mg}$ II absorption to a sensitive upper limit.

We examine the correlation between the properties of galaxies and $\mathrm{Mg} \mathrm{II}$ absorption strengths, using the sample of 71 'isolated' galaxies. The main results of our study are summarized below.

1. Based on a likelihood analysis, we confirm previous results that the Mg II absorber strength $W_{r}(2796)$ declines with increasing galaxy impact parameter and that the $W_{r}(2796)$ vs. $\rho$ anti-correlation is further strengthened after accounting for the scaling relation with galaxy $B$-band luminosity. The significantly improved anti-correlation indicates that more luminous galaxies are surrounded by more extended $\mathrm{Mg}$ II halos.

2. While galaxies from a 'group' environment appear to occupy a similar $W_{r}(2796)$ versus $\rho$ parameter space with 'isolated' galaxies, they do not exhibit a strong inverse correlation.

3. Including intrinsic $B_{A B}-R_{A B}$ color does not improve the observed $W_{r}(2796)$ vs. $\rho$ anti-correlation. The broad range of intrinsic colors in our sample, indicating a broad range of galaxy types from E/SO to starburst, shows that extended $\mathrm{Mg}$ II halos are a common and generic feature of ordinary galaxies. It suggests a lack of physical connection between the origin of extended $\mathrm{Mg}$ II halos and recent star formation history of the galaxies.

4. Including galaxy redshift does not improve the $W_{r}$ (2796) vs. $\rho$ anti-correlation. Therefore, our galaxy sample reveals little evolution in the extended Mg II halos between $z \approx 0.5$ and $z \approx 0.1$.

5. The extended halos of Mg II absorbing gas around ordinary galaxies at $z \sim 0.25$ are best described by $R_{\mathrm{gas}} / R_{\mathrm{gas}_{*}}=$ $\left(L_{B} / L_{B_{*}}\right)^{0.35 \pm 0.03}$ and $R_{\mathrm{gas}_{*}} \approx 75 h^{-1} \mathrm{kpc}$, indicating that gaseous radius is a constant fraction of halo radius over the mass scale of $M_{h}=10^{10.6}-10^{13} h^{-1} \mathrm{M}_{\odot}$ sampled in our observations. No correlation is found between the number density of cool gas clouds and galaxy luminosity.

6. The covering fraction of extended Mg II absorbing gas is high. Within $R_{\text {gas }}$, we find a mean covering fraction of $\left\langle\kappa_{0.3}\right\rangle \approx 70 \%$ for absorbers of $W_{r}(2796) \geq 0.3 \AA$ and $\left\langle\kappa_{0.1}\right\rangle \approx$ $80 \%$ for absorbers of $W_{r}(2796) \geq 0.1 \AA$.

7. Interpreting the remaining scatter in the luminosityscaled $W_{r}(2796)$ versus $\rho$ anti-correlation as due to Poisson noise in the number of intercepted absorbing clumps along the sightline, we estimate that a sightline at $\langle\rho\rangle=26 h^{-1} \mathrm{kpc}$ intercepts on average a total of $n^{\text {clump }} \sim 18$ clumps through the gaseous halo. Adopting an isothermal density distribution of the clumps, we further estimate a total baryonic mass of the cool clumps probed by $\mathrm{Mg}$ II features of $M_{b} \sim$ $(3-6) \times 10^{9} h^{-2} \mathrm{M}_{\odot}$. The inferred total mass would be still larger if other factors contribute to the observed scatter in the mean $W_{r}(2796)$ versus $\rho$ relation.

The results from our survey are consistent with previous studies based on smaller samples. The large covering fraction of $\mathrm{Mg}$ II absorbing gas and the scaling relation between gaseous extent and galaxy $B$-band luminosity provide the empirical support for the halo occupation model developed in Tinker \& Chen $(2008,2009)$ and in Gauthier et al. (2009).

We are grateful to the SDSS collaboration for producing and maintaining the SDSS public data archive. We thank Niraj Welikala for providing photometric measurements of galaxy SDSSJ090519.72+084914.02. We also thank 
Betsy Barton, Nick Gnedin, Andrey Kravtsov, and Mary Putman for helpful discussions. H.-W.C. acknowledges partial support from NASA Long Term Space Astrophysics grant NNG06GC36G and an NSF grant AST-0607510. J.-R.G. acknowledges partial support from a Brinson Foundation Predoctoral Fellowship. Funding for the SDSS and SDSS-II was provided by the Alfred P. Sloan Foundation, the Participating
Institutions, the National Science Foundation, the U.S. Department of Energy, the National Aeronautics and Space Administration, the Japanese Monbukagakusho, the Max Planck Society, and the Higher Education Funding Council for England. The SDSS was managed by the Astrophysical Research Consortium for the Participating Institutions.

\section{REFERENCES}

Balogh, M. L., Navarro, J. F., \& Morris, S. L. 2000, ApJ, 540, 113

Barton, E. J. \& Cooke, J. 2009, AJ, 138, 1817

Bechtold, J. et al. 2002, ApJS, 140, 138

Bergeron, J. 1986, A\&A, 155, L8

Bergeron, J. \& Stasínska, G. 1986, A\&A, 169, 1

Berlind, A. A., \& Weinberg, D. H. 2002, ApJ, 575, 587

Binney, J., Dehnen, W., \& Gianpaolo, B. 2000, MNRAS, 318, 658

Birnboim, Y. \& Dekel, A. 2003, MNRAS, 345, 349

Birnboim, Y., Dekel, A., \& Neistein, E. 2007, MNRAS, 380, 339

Blake, C., Collister, A., \& Lahav, O. 2008, MNRAS, 385, 1257

Bond, N. A., Churchill, C. W., \& Charlton, J. C. 2001, ApJ, 562, 641

Bowen, D. V., Blades, J. C., \& Pettini, M. 1995, ApJ, 448, 634

Bouché, N., Murphy, M. T., Péroux, C., Csabai, I., \& Wild, V. 2006, MNRAS, 371,495

Bregman, J. N. 1980, ApJ, 236, 577

Charlton, J. C. \& Churchill, C. W. 1998, ApJ, 499, 181

Charlton, J. C., Ding, J., Zonak, S. G., Churchill, C. W., Bond, N. A., \& Rigby, J. R. 2003, ApJ, 589, 111

Chelouche, D., Ménard, B., Bowen, D. V., \& Gnat, O. 2007, ApJ in press, (astro-ph/0706.4336)

Chen, H.-W., Lanzetta, K. M., \& Webb, J. K. 2001, ApJ, 556, 158

Chen, H.-W., Kennicutt, R. C. Jr., \& Rauch, M. 2005, ApJ, 620, 703

Chen, H.-W. \& Tinker, J. L. 2008, ApJ, 687, 745

Chen, H.-W. \& Mulchaey, J. S. 2009, ApJ, 701, 1219

Churchill, C. W., Mellon, R. R., Charlton, J. C., Jannuzi, B. T., Kirhakos, S., Steidel, C. C. \& Schneider, D. P. 2000, ApJS, 130,91

Churchill, C. W., Kacprzak, G. G., Steidel, C. C., \& Evans, J. L. 2007, ApJ, 661,714

Coleman, G. D., Wu, C. C., \& Weedman, D. W. 1980, ApJS, 43, 393

Dekel, A. \& Birnboim, Y. 2006, MNRAS, 368, 2

Dolag, K., Bartelmann, M., Perrotta, F., Baccigalupi, C., Moscardini, L., Meneghetti, M., \& Tormen, G. 2004, A\&A, 416, 853

Doyle, M. T. et al. 2005, MNRAS, 361, 34

Faber, S. M. et al. 2007, ApJ, 665, 265

Feigelson, E. D. \& Nelson, P. I. 1985, ApJ, 293, 192

Fukugita, M., Shimasaku, K., \& Ichikawa, T. 1995, PASP, 107, 945

Gauthier, J.-R., Chen, H.-W., \& Tinker, J. L. 2009, ApJ, 702, 50

Gauthier, J.-R., Chen, H.-W., \& Tinker, J. L. 2010, ApJ submitted

Gunn, J. E. \& Gott, J. R. III 1972, ApJ, 176, 1

Heitsch, F. \& Putman, M. E. 2009, ApJ, 698, 1485

Jannuzi, B. T. et al. 1998, ApJS, 118, 1

Kacprzak, G. G., Churchill, C. W., Steidel, C. C., Murphy, M. T., \& Evans, J. L. 2007, ApJ, 662, 909

Kacprzak, G. G., Churchill, C. W., Steidel, C. C., \& Murphy, M. T. 2008, AJ, 135,922

Kacprzak, G. G., Churchill, C. W., Ceverino, D., Steidel, C. C., Klypin, A., \& Murphy, M. T. 2010, ApJ, 711, 533

Kaufmann, T., Bullock, J. S., Maller, A. H., Fan, T., \& Wadsley, J. 2009, MNRAS, 396, 191
Kereš, D., Katz, N., Weinberg, D. H., \& Davé, R. 2005, MNRAS, 363, 2 Kereš, D., Katz, N., Fardel, M., Davé, R., \& Weinberg, D. H. 2009, MNRAS, 395,160

Kereš, D. \& Hernquist, L. 2009, ApJ, 700, L1

Lanzetta, K. M., Wolfe, A. M., \& Turnshek, D. A. 1987, ApJ, 322, 739

Lanzetta, K. M. \& Bowen, D. 1990, ApJ, 357, 321

Lundgren, B. F. et al. 2009, ApJ, 698, 819

Lupton, R. 1995, http://www.apo.nmsu.edu/35m operations/35m_manual/Instruments/DIS/[

Maller, A. H. \& Bullock, J. S. 2004, MNRAS, 355, 694

Marshall, J. L. et al. 2008, Proc. SPIE, 7014, 169

McGaugh, S. S., Schombert, J. M., de Blok, W. J. G., \& Zagursky, M. J. 2010, ApJ, 708, L14

Mo, H. J. \& Miralda-Escudé, J. 1996, ApJ, 469, 589

Navarro, J. F., Frenk, C. S., \& White, S. D. M. 1996, ApJ, 490, 493

Nestor, D. B., Turnshek, D. A., \& Rao, S. M. 2005, ApJ, 628, 637

Nestor, D. B., Turnshek, D. A., Rao, S. M., \& Quider, A. M. 2007, ApJ, 658, 185

Petitjean, P. \& Bergeron, J. 1990, A\&A, 231, 309

Prochter, G. E., Prochaska, J. X., \& Burles, S. M. 2006, ApJ, 639, 766

Putman, M. E. 2006, ApJ, 645, 1164

Rao, S. M., Turnshek, D. A., \& Nestor, D. B. 2006, ApJ, 636, 610

Robitaille, T. P. \& Whitney, B. A. 2010, ApJ, 710, L11

Sargent, W. L. W., Steidel, C. C., \& Boksenberg, A. 1988, ApJ, 334, 22

Schneider, D. P. et al. 2007, AJ, 134, 102

Scoccimarro, R., Sheth, R. K., Hui, L., \& Jain, B. 2001, ApJ, 546, 20

Seljak, U. 2000, MNRAS, 318, 203

Steidel, C. C. \& Sargent, W. L. W. 1992, ApJS, 80, 1

Steidel, C. C., Dickinson, M., \& Persson, S. E. 1994, ApJL, 437, L75

Steidel, C.C., Kollmeier, J.A., Shapely, A.E., Churchill, C.W., Dickinson, M., \& Pettini, M. 2002, ApJ, 570, 526

Thom, C., Peek, J. E. G., Putman, M. E., Heiles, C., Peek, K. M. G., \& Wilhelm, R. 2008, ApJ, 684, 364

Tinker, J. L., Norberg, P., Weinberg, D. H., \& Warren, M. S. 2007, ApJ, 659, 877

Tinker, J. L. \& Chen, H.-W. 2008, ApJ, 679, 1218

Tinker, J. L. \& Chen, H.-W. 2010, ApJ, 709,

Tripp, T. M. \& Bowen, D. V. 2005, in IAU Colloq. 199: Probing Galaxies through Quasar Absorption Lines, ed. P. Williams, C.-G. Shu, \& B. Menard, 5-23

Verdes-Montenegro, L., Yun, M., Williams, B. A., Huchmeier, W. K., Del Olmo, A., \& Perea, J. 2001, A\&A, 377, 812

Wild, V. et al. 2008, MNRAS, 388, 227

Westmeier, T., Brüns, C. \& Kerp, J. 2008, MNRAS, 390, 1691

York, D. G. et al. 2000, AJ, 120, 1579

Zheng, Z., Coil, A. L., \& Zehavi, I. 2007, ApJ, 667, 760 (ZCZ07)

Zibetti, S., Ménard, B., Nestor, D., \& Turnshek, D. 2005, ApJL, 631, L105

Zibetti, S., Ménard, B., Nestor, D., Quider, A. M., Rao, S. M., \& Turnshek, D. A. $2007, \mathrm{ApJ}, 658,161$ 
TABLE 1

Summary of Faint Galaxy Spectroscopy

\begin{tabular}{|c|c|c|c|c|c|c|c|}
\hline ID & $\mathrm{RA}(\mathrm{J} 2000)$ & $\operatorname{Dec}(\mathrm{J} 2000)$ & $z_{\text {phot }}$ & $r^{\prime}$ & Instrument & Exptime & UT Date \\
\hline SDSSJ003339.66-005518.36 & $00: 33: 39.66$ & $-00: 55: 18.36$ & $0.18 \pm 0.06$ & 19.6 & DIS & $3 \times 1200$ & $2008-11-23$ \\
\hline SDSSJ003339.85-005522.36 & $00: 33: 39.85$ & $-00: 55: 22.36$ & $0.16 \pm 0.03$ & 18.8 & MagE & $2 \times 300$ & 2008-09-23 \\
\hline SDSSJ003341.47-005522.79 & $00: 33: 41.47$ & $-00: 55: 22.79$ & $0.28 \pm 0.12$ & 20.5 & DIS & $2 \times 1800+1200$ & $2008-10-24$ \\
\hline SDSSJ003407.78-085453.28 & $00: 34: 07.78$ & $-08: 54: 53.28$ & $0.35 \pm 0.12$ & 21.3 & MagE & 600 & 2008-09-24 \\
\hline SDSSJ003412.85-010019.79 & $00: 34: 12.85$ & $-01: 00: 19.79$ & $0.25 \pm 0.05$ & 20.1 & MagE & $2 \times 900$ & 2008-09-23 \\
\hline SDSSJ003414.49-005927.49 & $00: 34: 14.49$ & $-00: 59: 27.49$ & $0.12 \pm 0.01$ & 17.2 & SDSS & & \\
\hline SDSSJ010136.52-005016.44 & 01:01:36.52 & $-00: 50: 16.44$ & $0.22 \pm 0.06$ & 19.4 & MagE & $2 \times 600$ & 2008-09-24 \\
\hline SDSSJ010155.80-084408.74 & 01:01:55.80 & $-08: 44: 08.74$ & $0.26 \pm 0.10$ & 20.1 & MagE & 600 & $2008-09-25$ \\
\hline SDSSJ010351.82+003740.77 & 01:03:51.82 & $+00: 37: 40.77$ & $0.29 \pm 0.13$ & 21.3 & MagE & $2 \times 600$ & 2008-09-25 \\
\hline SDSSJ021558.84-011131.23 & $02: 15: 58.84$ & $-01: 11: 31.23$ & $0.16 \pm 0.07$ & 19.3 & DIS & $2 \times 1800+1300$ & 2008-11-01 \\
\hline SDSSJ022949.97-074255.88 & $02: 29: 49.97$ & $-07: 42: 55.88$ & $0.27 \pm 0.17$ & 20.9 & DIS & $3 \times 1800$ & $2008-11-30$ \\
\hline SDSSJ024127.75-004517.04 & $02: 41: 27.75$ & $-00: 45: 17.04$ & $0.14 \pm 0.04$ & 18.6 & DIS & $1800+1500$ & 2008-11-17 \\
\hline SDSSJ032230.27+003712.72 & $03: 22: 30.27$ & $+00: 37: 12.72$ & $0.20 \pm 0.03$ & 17.5 & DIS & $2 \times 900$ & $2008-11-23$ \\
\hline SDSSJ032232.55+003644.68 & $03: 22: 32.55$ & $+00: 36: 44.68$ & $0.38 \pm 0.12$ & 21.2 & DIS & $2 \times 1800$ & $2008-12-22$ \\
\hline SDSSJ035241.99+001317.13 & 03:52:41.99 & $+00: 13: 17.13$ & $0.28 \pm 0.08$ & 20.3 & MagE & $2 \times 600$ & $2008-09-24$ \\
\hline SDSSJ040404.51-060709.46 & 04:04:04.51 & $-06: 07: 09.46$ & $0.32 \pm 0.10$ & 20.2 & MagE & 1200 & $2009-02-22$ \\
\hline SDSSJ074527.07+191959.90 & 07:45:27.07 & $+19: 19: 59.90$ & $0.36 \pm 0.03$ & 19.7 & DIS & $2 \times 1800$ & 2009-02-18 \\
\hline SDSSJ074527.22+192003.88 & $07: 45: 27.22$ & $+19: 20: 03.88$ & $0.28 \pm 0.09$ & 20.2 & DIS & $2 \times 1800$ & 2009-02-18 \\
\hline SDSSJ075001.34+161301.92 & 07:50:01.34 & $+16: 13: 01.92$ & $0.17 \pm 0.11$ & 20.6 & MagE & $300+600$ & 2009-03-28 \\
\hline SDSSJ075450.11+185005.28 & 07:54:50.11 & $+18: 50: 05.28$ & $0.33 \pm 0.04$ & 19.7 & DIS & $2 \times 1200$ & $2009-02-18$ \\
\hline SDSSJ075525.13+172825.79 & $07: 55: 25.13$ & $+17: 28: 25.79$ & $0.18 \pm 0.07$ & 19.3 & DIS & $2 \times 1200$ & 2009-02-18 \\
\hline SDSSJ080005.11+184933.31 & 08:00:05.11 & $+18: 49: 33.31$ & $0.28 \pm 0.08$ & 19.9 & DIS & 1800 & 2009-03-19 \\
\hline SDSSJ082340.56+074751.07 & $08: 23: 40.56$ & $+07: 47: 51.07$ & $0.18 \pm 0.04$ & 18.5 & DIS & 1800 & 2009-01-05 \\
\hline SDSSJ083218.55+043337.81 & $08: 32: 18.55$ & $+04: 33: 37.81$ & $0.17 \pm 0.04$ & 18.1 & DIS & 1200 & 2009-03-19 \\
\hline SDSSJ083218.77+043346.58 & 08:32:18.77 & $+04: 33: 46.58$ & $0.17 \pm 0.02$ & 17.4 & DIS & 1200 & 2009-03-19 \\
\hline SDSSJ083221.60+043359.74 & $08: 32: 21.60$ & $+04: 33: 59.74$ & $0.16 \pm 0.08$ & 19.4 & DIS & $2 \times 1200$ & $2009-02-18$ \\
\hline SDSSJ084120.59+012628.85 & $08: 41: 20.59$ & $+01: 26: 28.85$ & $0.20 \pm 0.11$ & 20.0 & DIS & $2 \times 600$ & $2009-02-18$ \\
\hline SDSSJ084455.58+004718.15 & $08: 44: 55.58$ & $+00: 47: 18.15$ & $0.17 \pm 0.06$ & 18.8 & DIS & 1800 & 2009-01-05 \\
\hline SDSSJ085829.88+022616.04 & 08:58:29.88 & $+02: 26: 16.04$ & $0.12 \pm 0.04$ & 18.3 & DIS & 1800 & 2009-03-26 \\
\hline SDSSJ090519.01+084933.70 & 09:05:19.01 & $+08: 49: 33.70$ & $0.36 \pm 0.09$ & 20.3 & DIS & 1800 & 2009-03-19 \\
\hline SDSSJ090519.61+084932.22 & 09:05:19.61 & $+08: 49: 32.22$ & $0.27 \pm 0.18$ & 20.9 & MagE & 1200 & $2009-02-22$ \\
\hline SDSSJ090519.72+084914.02 & 09:05:19.72 & $+08: 49: 14.02$ & & 22.2 & MagE & $2 \times 1800$ & 2009-02-22 \\
\hline SDSSJ091845.10+060202.93 & 09:18:45.10 & $+06: 02: 02.93$ & $0.19 \pm 0.04$ & 18.6 & MagE & $600+3 \times 300$ & 2009-03-29 \\
\hline SDSSJ091845.70+060220.57 & $09: 18: 45.70$ & $+06: 02: 20.57$ & $0.37 \pm 0.13$ & 19.9 & MagE & 600 & $2009-03-28$ \\
\hline SDSSJ093252.25+073731.59 & $09: 32: 52.25$ & $+07: 37: 31.59$ & $0.35 \pm 0.11$ & 20.1 & MagE & 600 & 2009-03-29 \\
\hline SDSSJ093537.25+112410.66 & $09: 35: 37.25$ & $+11: 24: 10.66$ & $0.24 \pm 0.08$ & 20.4 & MagE & 600 & 2009-03-29 \\
\hline SDSSJ100810.61+014446.17 & $10: 08: 10.61$ & $+01: 44: 46.17$ & $0.17 \pm 0.03$ & 17.7 & DIS & 900 & 2009-03-26 \\
\hline SDSSJ100906.91+023557.81 & 10:09:06.91 & $+02: 35: 57.81$ & $0.29 \pm 0.07$ & 19.2 & MagE & 300 & $2009-03-28$ \\
\hline SDSSJ102220.71+013143.50 & $10: 22: 20.71$ & $+01: 31: 43.50$ & $0.12 \pm 0.01$ & 17.4 & SDSS & & \\
\hline SDSSJ103605.26+015654.88 & $10: 36: 05.26$ & $+01: 56: 54.88$ & $0.31 \pm 0.06$ & 18.8 & DIS & 1800 & 2009-03-19 \\
\hline SDSSJ103836.38+095143.68 & $10: 38: 36.38$ & $+09: 51: 43.68$ & $0.38 \pm 0.12$ & 20.3 & MagE & 600 & 2009-03-29 \\
\hline SDSSJ112016.63+093317.94 & $11: 20: 16.63$ & $+09: 33: 17.94$ & $0.34 \pm 0.11$ & 20.5 & MagE & 600 & $2009-03-28$ \\
\hline SDSSJ113756.76+085022.38 & $11: 37: 56.76$ & $+08: 50: 22.38$ & $0.35 \pm 0.11$ & 20.7 & MagE & 600 & 2009-03-29 \\
\hline SDSSJ114144.83+080554.09 & $11: 41: 44.83$ & $+08: 05: 54.09$ & $0.17 \pm 0.09$ & 19.3 & DIS & 1800 & 2009-03-19 \\
\hline SDSSJ114145.14+080605.27 & $11: 41: 45.14$ & $+08: 06: 05.27$ & $0.34 \pm 0.07$ & 20.0 & DIS & 1800 & 2009-03-19 \\
\hline SDSSJ114830.94+021807.91 & $11: 48: 30.94$ & $+02: 18: 07.91$ & $0.33 \pm 0.04$ & 19.8 & DIS & 1200 & $2009-03-26$ \\
\hline SDSSJ114831.01+021803.00 & $11: 48: 31.01$ & $+02: 18: 03.00$ & $0.29 \pm 0.05$ & 19.6 & DIS & 1200 & 2009-03-26 \\
\hline SDSSJ120931.61+004546.23 & 12:09:31.61 & $+00: 45: 46.23$ & $0.30 \pm 0.09$ & 20.5 & MagE & $2 \times 1200$ & $2009-02-22$ \\
\hline SDSSJ121347.09+000141.26 & $12: 13: 47.09$ & $+00: 01: 41.26$ & $0.26 \pm 0.09$ & 20.2 & MagE & 1200 & $2009-06-20$ \\
\hline SDSSJ121347.14+000136.62 & $12: 13: 47.14$ & $+00: 01: 36.62$ & $0.26 \pm 0.06$ & 19.1 & SDSS & & \\
\hline SDSSJ122115.84-020259.37 & $12: 21: 15.84$ & $-02: 02: 59.37$ & $0.25 \pm 0.16$ & 20.6 & MagE & 600 & 2008-03-29 \\
\hline SDSSJ125737.93+144802.20 & $12: 57: 37.93$ & $+14: 48: 02.20$ & $0.30 \pm 0.09$ & 20.3 & MagE & 600 & 2009-03-29 \\
\hline SDSSJ130555.49+014928.62 & 13:05:55.49 & $+01: 49: 28.62$ & $0.32 \pm 0.06$ & 19.2 & DIS & 1800 & 2009-04-01 \\
\hline SDSSJ130557.05+014922.34 & 13:05:57.05 & $+01: 49: 22.34$ & $0.17 \pm 0.01$ & 17.7 & DIS & 1800 & 2009-04-01 \\
\hline SDSSJ132757.22+101136.02 & $13: 27: 57.22$ & $+10: 11: 36.02$ & $0.37 \pm 0.13$ & 20.5 & MagE & 600 & 2009-03-28 \\
\hline SDSSJ132829.30+080003.17 & $13: 28: 29.30$ & $+08: 00: 03.17$ & $0.20 \pm 0.07$ & 19.3 & DIS & 1800 & 2009-04-01 \\
\hline SDSSJ132830.62+080005.22 & $13: 28: 30.62$ & $+08: 00: 05.22$ & $0.22 \pm 0.05$ & 19.0 & DIS & 1800 & 2009-04-01 \\
\hline SDSSJ132831.15+075923.90 & $13: 28: 31.15$ & $+07: 59: 23.90$ & $0.35 \pm 0.08$ & 20.4 & DIS & 1800 & 2009-04-01 \\
\hline SDSSJ132831.54+075943.00 & $13: 28: 31.54$ & $+07: 59: 43.00$ & $0.35 \pm 0.09$ & 19.5 & MagE & 300 & 2009-03-29 \\
\hline SDSSJ132832.74+075952.56 & $13: 28: 32.74$ & $+07: 59: 52.56$ & $0.19 \pm 0.09$ & 19.3 & DIS & 1800 & 2009-04-01 \\
\hline SDSSJ133905.86+002225.36 & $13: 39: 05.86$ & $+00: 22: 25.36$ & $0.14 \pm 0.01$ & 17.5 & SDSS & & \\
\hline SDSSJ140618.34+130143.61 & $14: 06: 18.34$ & $+13: 01: 43.61$ & $0.14 \pm 0.05$ & 18.0 & DIS & 1200 & 2009-04-01 \\
\hline SDSSJ140619.94+130105.23 & 14:06:19.94 & $+13: 01: 05.23$ & $0.23 \pm 0.09$ & 19.7 & MagE & 300 & 2009-03-29 \\
\hline SDSSJ142600.05-001818.12 & $14: 26: 00.05$ & $-00: 18: 18.12$ & $0.14 \pm 0.01$ & 15.9 & SDSS & & \\
\hline SDSSJ143216.97+095522.23 & $14: 32: 16.97$ & $+09: 55: 22.23$ & $0.37 \pm 0.07$ & 20.5 & MagE & 600 & 2009-03-28 \\
\hline SDSSJ150339.62+064235.04 & $15: 03: 39.62$ & $+06: 42: 35.04$ & $0.22 \pm 0.10$ & 20.3 & DIS & 1800 & 2009-04-01 \\
\hline SDSSJ150340.15+064308.11 & $15: 03: 40.15$ & $+06: 43: 08.11$ & $0.30 \pm 0.11$ & 20.5 & MagE & $300+600$ & 2009-03-29 \\
\hline SDSSJ151228.25-011216.09 & $15: 12: 28.25$ & $-01: 12: 16.09$ & $0.16 \pm 0.03$ & 19.0 & MagE & 600 & $2009-02-23$ \\
\hline SDSSJ153112.77+091119.72 & $15: 31: 12.77$ & $+09: 11: 19.72$ & $0.17 \pm 0.11$ & 20.8 & MagE & 600 & $2009-03-28$ \\
\hline SDSSJ153113.01+091127.02 & $15: 31: 13.01$ & $+09: 11: 27.02$ & $0.22 \pm 0.11$ & 20.6 & MagE & 600 & 2009-03-28 \\
\hline SDSSJ153715.67+023056.39 & $15: 37: 15.67$ & $+02: 30: 56.39$ & $0.23 \pm 0.09$ & 19.5 & MagE & 900 & 2009-06-20 \\
\hline SDSSJ155336.77+053438.23 & $15: 53: 36.77$ & $+05: 34: 38.23$ & $0.24 \pm 0.06$ & 19.2 & DIS & 1800 & 2009-04-02 \\
\hline SDSSJ155556.54-003615.58 & $15: 55: 56.54$ & $-00: 36: 15.58$ & $0.24 \pm 0.15$ & 20.9 & MagE & 600 & 2009-03-29 \\
\hline SDSSJ160749.54-002228.42 & $16: 07: 49.54$ & $-00: 22: 28.42$ & $0.32 \pm 0.09$ & 19.6 & MagE & 900 & 2009-06-20 \\
\hline SDSSJ160906.36+071330.66 & 16:09:06.36 & $+07: 13: 30.66$ & $0.18 \pm 0.05$ & 18.9 & MagE & $2 \times 300$ & 2009-03-28 \\
\hline SDSSJ204303.53-010139.05 & $20: 43: 03.53$ & $-01: 01: 39.05$ & $0.19 \pm 0.04$ & 18.7 & DIS & $2 \times 1800$ & $2008-10-24$ \\
\hline & $20: 43: 04.34$ & $-01: 01: 37.91$ & $0.13 \pm 0.07$ & 19.5 & DIS & $2 \times 1800$ & $2008-10-24$ \\
\hline SDSSJ204431.32+011304.97 & $20: 44: 31.32$ & $+01: 13: 04.97$ & $0.16 \pm 0.04$ & 18.7 & MagE & $300+2 \times 600$ & $2008-09-24$ \\
\hline SDSSJ204431.87+011308.81 & $20: 44: 31.87$ & $+01: 13: 08.81$ & $0.19 \pm 0.11$ & 20.4 & DIS & 1800 & 2008-11-17 \\
\hline & & & & & & & \\
\hline
\end{tabular}


Extended Cool Gas around Galaxies

TABLE 2

SuMmary OF THE MAGE SPECTROSCOPIC OBSERVATIONS OF SDSS QSOS

\begin{tabular}{|c|c|c|c|c|c|c|}
\hline ID & RA(J2000) & $\operatorname{Dec}(J 2000)$ & zQSO & $u^{\prime}$ & Exptime & UT Date \\
\hline SDSSJ003340.21-005525.53 & $00: 33: 40.21$ & $-00: 55: 25.53$ & 0.94 & 17.99 & $3 \times 900$ & $2008-09-23$ \\
\hline SDSSJ003407.34-085452.07 & $00: 34: 07.34$ & $-08: 54: 52.07$ & 1.31 & 18.59 & $2 \times 1200$ & 2008-09-24 \\
\hline SDSSJ003413.04-010026.86 & $00: 34: 13.04$ & $-01: 00: 26.86$ & 1.29 & 17.33 & $2 \times 600$ & $2008-09-23$ \\
\hline SDSSJ010135.84-005009.08 & $01: 01: 35.84$ & $-00: 50: 09.08$ & 1.01 & 19.31 & $2 \times 1800$ & $2008-09-24$ \\
\hline SDSSJ010156.32-084401.74 & $01: 01: 56.32$ & $-08: 44: 01.74$ & 0.98 & 18.29 & $2 \times 1800$ & $2008-09-25$ \\
\hline SDSSJ010352.47+003739.79 & $01: 03: 52.47$ & $+00: 37: 39.79$ & 0.70 & 18.36 & $3 \times 1200$ & $2008-09-25$ \\
\hline SDSSJ010508.14-005041.33 & 01:05:08.14 & $-00: 50: 41.33$ & 1.59 & 18.28 & $2 \times 1200$ & 2008-09-25 \\
\hline SDSSJ021558.40-011135.79 & $02: 15: 58.40$ & $-01: 11: 35.79$ & 0.84 & 17.85 & $2 \times 1200$ & $2008-09-23$ \\
\hline SDSSJ022950.32-074256.77 & $02: 29: 50.32$ & $-07: 42: 56.77$ & 1.56 & 19.16 & $900+1200$ & $2008-09-25$ \\
\hline SDSSJ024126.71-004526.25 & $02: 41: 26.71$ & $-00: 45: 26.25$ & 0.72 & 18.36 & $2 \times 900$ & $2008-09-24$ \\
\hline SDSSJ032232.58+003649.13 & $03: 22: 32.58$ & $+00: 36: 49.13$ & 1.59 & 19.50 & $2 \times 1200$ & 2008-09-25 \\
\hline SDSSJ035242.12+001307.32 & $03: 52: 42.12$ & $+00: 13: 07.32$ & 1.16 & 19.21 & $1400+1800$ & $2008-09-24$ \\
\hline SDSSJ040404.08-060714.03 & 04:04:04.08 & $-06: 07: 14.03$ & 1.29 & 19.00 & $2 \times 1800$ & $2009-02-22$ \\
\hline SDSSJ074528.15+191952.68 & $07: 45: 28.15$ & $+19: 19: 52.68$ & 0.69 & 17.95 & $2 \times 1800$ & $2009-02-22$ \\
\hline SDSSJ075001.85+161305.05 & 07:50:01.85 & $+16: 13: 05.05$ & 1.10 & 18.41 & $2 \times 1800$ & $2009-03-28$ \\
\hline SDSSJ075450.04+184952.79 & $07: 54: 50.04$ & $+18: 49: 52.79$ & 0.81 & 18.37 & $2 \times 1800$ & $2009-02-23$ \\
\hline SDSSJ075525.51+172836.59 & $07: 55: 25.51$ & $+17: 28: 36.59$ & 1.29 & 18.38 & $2 \times 1800$ & $2009-02-22$ \\
\hline SDSSJ080004.56+184935.15 & 08:00:04.56 & $+18: 49: 35.15$ & 1.29 & 17.99 & $1500+1800$ & $2009-03-29$ \\
\hline SDSSJ082340.18+074801.68 & $08: 23: 40.18$ & $+07: 48: 01.68$ & 0.84 & 18.33 & $2 \times 1800$ & $2009-03-28$ \\
\hline SDSSJ083220.74+043416.78 & $08: 32: 20.74$ & $+04: 34: 16.78$ & 1.51 & 18.25 & $3 \times 1800$ & $2008-02-03$ \\
\hline SDSSJ084119.78+012621.75 & $08: 41: 19.78$ & $+01: 26: 21.75$ & 1.48 & 18.20 & $2 \times 1800$ & $2008-02-02$ \\
\hline SDSSJ084456.06+004708.95 & $08: 44: 56.06$ & $+00: 47: 08.95$ & 1.31 & 18.45 & $2 \times 1800$ & $2009-02-23$ \\
\hline SDSSJ085826.93+022604.49 & $08: 58: 26.93$ & $+02: 26: 04.49$ & 1.51 & 18.19 & $1200+1800$ & $2008-02-02$ \\
\hline SDSSJ090519.70+084917.32 & 09:05:19.70 & $+08: 49: 17.32$ & 1.43 & 18.45 & $2 \times 1800$ & $2009-02-22$ \\
\hline SDSSJ091845.91+060226.09 & $09: 18: 45.91$ & $+06: 02: 26.09$ & 0.79 & 18.31 & $2 \times 1800$ & $2009-03-28$ \\
\hline SDSSJ093251.82+073729.11 & $09: 32: 51.82$ & $+07: 37: 29.11$ & 0.90 & 17.87 & $2 \times 1800$ & 2009-03-29 \\
\hline SDSSJ093536.98+112408.03 & $09: 35: 36.98$ & $+11: 24: 08.03$ & 0.85 & 18.30 & $600+1800$ & $2009-03-29$ \\
\hline SDSSJ100807.51+014448.97 & 10:08:07.51 & $+01: 44: 48.97$ & 1.33 & 18.25 & $2 \times 1800$ & $2009-02-23$ \\
\hline SDSSJ100906.36+023555.31 & $10: 09: 06.36$ & $+02: 35: 55.31$ & 1.10 & 17.20 & $2 \times 1200$ & $2009-03-28$ \\
\hline SDSSJ102218.98+013218.82 & $10: 22: 18.98$ & $+01: 32: 18.82$ & 0.79 & 16.92 & $900+1800$ & $2009-02-22$ \\
\hline SDSSJ103607.51+015659.14 & $10: 36: 07.51$ & $+01: 56: 59.14$ & 1.86 & 19.07 & $2 \times 1800$ & $2008-01-31$ \\
\hline SDSSJ103836.50+095138.85 & $10: 38: 36.50$ & $+09: 51: 38.85$ & 1.02 & 18.36 & $2 \times 1200$ & $2009-03-29$ \\
\hline SDSSJ112016.66+093323.53 & $11: 20: 16.66$ & $+09: 33: 23.53$ & 1.10 & 18.02 & $2 \times 1800$ & $2009-03-28$ \\
\hline SDSSJ113757.02+085017.21 & $11: 37: 57.02$ & $+08: 50: 17.21$ & 1.10 & 18.38 & 1200 & 2009-03-29 \\
\hline SDSSJ114144.62+080614.79 & $11: 41: 44.62$ & $+08: 06: 14.79$ & 1.08 & 18.37 & $1500+1800$ & $2009-02-23$ \\
\hline SDSSJ114830.12+021829.78 & $11: 48: 30.12$ & $+02: 18: 29.78$ & 1.22 & 18.00 & $2 \times 1700$ & 2008-01-31 \\
\hline SDSSJ120932.26+004555.92 & $12: 09: 32.26$ & $+00: 45: 55.92$ & 1.44 & 18.42 & $2 \times 1800$ & $2009-02-22$ \\
\hline SDSSJ121347.52+000129.99 & $12: 13: 47.52$ & $+00: 01: 29.99$ & 0.96 & 18.35 & $2 \times 1800$ & $2009-06-21$ \\
\hline SDSSJ122115.34-020253.39 & $12: 21: 15.34$ & $-02: 02: 53.39$ & 0.79 & 17.82 & 1200 & $2009-03-29$ \\
\hline SDSSJ125739.22+144806.26 & $12: 57: 39.22$ & $+14: 48: 06.26$ & 0.82 & 18.34 & 1200 & 2009-03-29 \\
\hline SDSSJ130554.17+014929.82 & $13: 05: 54.17$ & $+01: 49: 29.82$ & 0.73 & 18.00 & $1200+1800$ & 2009-02-23 \\
\hline SDSSJ132757.41+101141.78 & $13: 27: 57.41$ & $+10: 11: 41.78$ & 1.37 & 18.14 & $2 \times 1200$ & $2009-03-28$ \\
\hline SDSSJ132831.08+075942.01 & $13: 28: 31.08$ & $+07: 59: 42.01$ & 1.33 & 18.26 & 1200 & $2009-03-29$ \\
\hline SDSSJ133904.34+002221.92 & $13: 39: 04.34$ & $+00: 22: 21.92$ & 1.15 & 17.98 & $1200+1800$ & 2009-06-21 \\
\hline SDSSJ140619.61+130106.82 & $14: 06: 19.61$ & $+13: 01: 06.82$ & 1.02 & 18.35 & 1200 & 2009-03-29 \\
\hline SDSSJ142556.40-001818.79 & $14: 25: 56.40$ & $-00: 18: 18.79$ & 1.15 & 18.96 & $1500+3000$ & $2008-07-24$ \\
\hline SDSSJ143216.78+095519.29 & $14: 32: 16.78$ & $+09: 55: 19.29$ & 0.77 & 17.67 & 1200 & $2009-03-28$ \\
\hline SDSSJ150339.98+064259.96 & $15: 03: 39.98$ & $+06: 42: 59.96$ & 0.94 & 18.26 & 1200 & 2009-03-29 \\
\hline SDSSJ151228.82-011223.12 & $15: 12: 28.82$ & $-01: 12: 23.12$ & 1.17 & 18.39 & $1200+1800$ & $2009-02-23$ \\
\hline SDSSJ153112.98+091138.78 & $15: 31: 12.98$ & $+09: 11: 38.78$ & 1.23 & 18.19 & $600+1200$ & $2009-03-28$ \\
\hline SDSSJ153715.34+023049.73 & $15: 37: 15.34$ & $+02: 30: 49.73$ & 0.48 & 17.65 & $2 \times 2400$ & $2009-06-20$ \\
\hline SDSSJ155336.46+053423.97 & $15: 53: 36.46$ & $+05: 34: 23.97$ & 1.14 & 18.38 & $2 \times 1800$ & $2009-06-21$ \\
\hline SDSSJ155557.07-003608.41 & $15: 55: 57.07$ & $-00: 36: 08.41$ & 0.76 & 18.22 & 1200 & 2009-03-29 \\
\hline SDSSJ160749.34-002219.86 & $16: 07: 49.34$ & $-00: 22: 19.86$ & 1.30 & 18.05 & $2 \times 1800$ & $2009-06-20$ \\
\hline SDSSJ160905.42+071337.29 & $16: 09: 05.42$ & $+07: 13: 37.29$ & 0.70 & 18.10 & $2 \times 1200$ & $2009-03-28$ \\
\hline SDSSJ204303.55-010126.05 & $20: 43: 03.55$ & $-01: 01: 26.05$ & 1.19 & 17.62 & $2 \times 1200$ & $2008-07-27$ \\
\hline SDSSJ204431.46+011312.43 & $20: 44: 31.46$ & $+01: 13: 12.43$ & 0.98 & 19.07 & $3 \times 1800$ & 2008-09-24 \\
\hline SDSSJ210230.72+094125.08 & $21: 02: 30.72$ & $+09: 41: 25.08$ & 0.79 & 19.19 & $2 \times 1200$ & $2008-09-24$ \\
\hline SDSSJ212938.59-063801.85 & $21: 29: 38.59$ & $-06: 38: 01.85$ & 1.01 & 18.08 & $900+1200$ & $2008-09-23$ \\
\hline SDSSJ221126.76+124458.16 & $22: 11: 26.76$ & $+12: 44: 58.16$ & 0.49 & 18.16 & $2 \times 900$ & $2008-09-23$ \\
\hline SDSSJ221526.74+011356.47 & $22: 15: 26.74$ & $+01: 13: 56.47$ & 1.26 & 19.21 & $2 \times 1200$ & $2008-09-23$ \\
\hline SDSSJ222849.20-005630.89 & $22: 28: 49.20$ & $-00: 56: 30.89$ & 1.28 & 18.96 & $900+1800$ & $2008-09-25$ \\
\hline SDSSJ223246.80+134702.04 & $22: 32: 46.80$ & $+13: 47: 02.04$ & 1.55 & 18.45 & $2 \times 1200$ & $2008-09-23$ \\
\hline SDSSJ223316.87+133309.90 & $22: 33: 16.87$ & $+13: 33: 09.90$ & 1.54 & 18.59 & $2 \times 1800$ & $2008-09-25$ \\
\hline SDSSJ223359.93-003315.79 & $22: 33: 59.93$ & $-00: 33: 15.79$ & 1.21 & 17.42 & $2 \times 1800$ & $2009-06-21$ \\
\hline SDSSJ224704.78-081617.54 & $22: 47: 04.78$ & $-08: 16: 17.54$ & 0.97 & 17.54 & $1200+3000$ & $2008-07-25$ \\
\hline SDSSJ230225.49-082154.12 & $23: 02: 25.49$ & $-08: 21: 54.12$ & 1.01 & 19.13 & $2 \times 1200$ & 2008-09-24 \\
\hline SDSSJ230845.60-091449.45 & $23: 08: 45.60$ & $-09: 14: 49.45$ & 0.89 & 19.27 & $3 \times 1200$ & $2008-09-25$ \\
\hline SDSSJ232812.91-090522.56 & $23: 28: 12.91$ & $-09: 05: 22.56$ & 1.11 & 17.71 & $600+2400$ & $2008-07-25$ \\
\hline SDSSJ234949.61+003535.39 & $23: 49: 49.61$ & $+00: 35: 35.39$ & 1.24 & 18.26 & $1200+1500$ & $2008-09-23$ \\
\hline
\end{tabular}


TABLE 3

Galaxies AND Absorption Systems

\begin{tabular}{|c|c|c|c|c|c|c|c|c|c|}
\hline \multicolumn{8}{|c|}{ Galaxies } & \multicolumn{2}{|c|}{ Absorption Systems } \\
\hline $\begin{array}{l}\text { ID } \\
\text { (1) }\end{array}$ & $\begin{array}{c}\Delta \alpha \\
(\operatorname{arcsec}) \\
(2)\end{array}$ & $\begin{array}{c}\Delta \delta \\
(\operatorname{arcsec}) \\
(3)\end{array}$ & $\begin{array}{l}z_{\mathrm{gal}} \\
(4)\end{array}$ & $\begin{array}{c}\rho \\
\left(h^{-1} \mathrm{kpc}\right) \\
(5)\end{array}$ & $\begin{array}{l}r^{\prime} \\
(6)\end{array}$ & $\begin{array}{c}M_{B} \\
-5 \log h \\
(7)\end{array}$ & $\begin{array}{c}B_{A B}-R_{A B} \\
\quad \text { (8) }\end{array}$ & $\begin{array}{c}z_{\text {abs }} \\
(9)\end{array}$ & $\begin{array}{c}W(2796)^{\mathrm{a}} \\
(\AA) \\
(10)\end{array}$ \\
\hline SDSSJ003339.85-005522.36 & -5.4 & 3.2 & 0.2124 & 15.2 & 18.82 & -20.11 & 0.25 & 0.2121 & $1.05 \pm 0.03$ \\
\hline SDSSJ003407.78-085453.28 & 6.5 & -1.2 & 0.3617 & 23.5 & 21.37 & -18.89 & 0.79 & 0.3616 & $0.48 \pm 0.05$ \\
\hline SDSSJ003412.85-010019.79 & -2.8 & 7.1 & 0.2564 & 21.3 & 20.16 & -18.94 & 1.11 & 0.2564 & $0.61 \pm 0.06$ \\
\hline SDSSJ003414.49-005927.49 & 21.7 & 59.4 & 0.1202 & 96.0 & 17.24 & -19.80 & 1.14 & 0.1202 & $<0.22$ \\
\hline SDSSJ010136.52-005016.44 & 10.2 & -7.4 & 0.2615 & 35.9 & 19.43 & -19.71 & 1.15 & 0.2615 & $<0.07$ \\
\hline SDSSJ010155.80-084408.74 & -7.7 & -7.0 & 0.1588 & 20.0 & 20.19 & -17.70 & 0.65 & 0.1586 & $0.36 \pm 0.03$ \\
\hline SDSSJ010351.82+003740.77 & -9.7 & 1.0 & 0.3515 & 34.0 & 21.38 & -18.85 & 0.88 & 0.3508 & $0.38 \pm 0.03$ \\
\hline SDSSJ021558.84-011131.23 & 6.6 & 4.6 & 0.2103 & 19.3 & 19.35 & -19.35 & 0.84 & 0.2108 & $0.77 \pm 0.05$ \\
\hline SDSSJ022949.97-074255.88 & -5.2 & 0.9 & 0.3866 & 19.5 & 20.94 & -19.47 & 0.62 & 0.3861 & $1.74 \pm 0.04$ \\
\hline SDSSJ024127.75-004517.04 & 15.6 & 9.2 & 0.1765 & 37.9 & 18.69 & -19.39 & 1.26 & $\ldots$ & $\ldots$ \\
\hline SDSSJ032230.27+003712.72 & -34.6 & 23.6 & 0.1833 & 90.4 & 17.55 & -21.02 & 0.55 & & \\
\hline SDSSJ032232.55+003644.68 & -0.4 & -4.5 & 0.2185 & 11.1 & 21.27 & -17.48 & 0.85 & 0.2183 & $1.31 \pm 0.12$ \\
\hline SDSSJ035241.99+001317.13 & -1.9 & 9.8 & 0.3671 & 35.9 & 20.37 & -19.87 & 0.72 & 0.3677 & $1.45 \pm 0.05$ \\
\hline SDSSJ040404.51-060709.46 & 6.4 & 4.6 & 0.2387 & 20.8 & 20.20 & -18.89 & 0.62 & & \\
\hline SDSSJ075001.34+161301.92 & -7.3 & -3.1 & 0.1466 & 14.3 & 20.65 & -17.19 & 0.66 & 0.1469 & $0.26 \pm 0.08$ \\
\hline SDSSJ075450.11+185005.28 & 1.0 & 12.5 & 0.2856 & 37.8 & 19.77 & -19.69 & 1.08 & 0.2856 & $<0.04$ \\
\hline SDSSJ075525.13+172825.79 & -5.4 & -10.8 & 0.2541 & 33.5 & 19.34 & -19.82 & 1.02 & 0.2546 & $0.51 \pm 0.02$ \\
\hline SDSSJ080005.11+184933.31 & 7.8 & -1.8 & 0.2544 & 22.2 & 19.94 & -19.33 & 0.48 & 0.2536 & $0.30 \pm 0.04$ \\
\hline SDSSJ082340.56+074751.07 & 5.6 & -10.6 & 0.1864 & 26.3 & 18.54 & -19.67 & 1.00 & 0.1863 & $0.37 \pm 0.04$ \\
\hline SDSSJ084120.59+012628.85 & 12.1 & 7.1 & 0.4091 & 53.7 & 20.05 & -20.54 & 0.81 & 0.4084 & $0.10 \pm 0.02$ \\
\hline SDSSJ084455.58+004718.15 & -7.2 & 9.2 & 0.1551 & 22.0 & 18.86 & -19.10 & 0.72 & 0.1554 & $0.40 \pm 0.05$ \\
\hline SDSSJ085829.88+022616.04 & 44.2 & 11.6 & 0.1097 & 64.0 & 18.34 & -18.81 & 0.75 & 0.1097 & $<0.06$ \\
\hline SDSSJ090519.01+084933.70 & -10.2 & 16.4 & 0.3856 & 71.1 & 20.33 & -20.07 & -0.13 & 0.3856 & $<0.04$ \\
\hline SDSSJ090519.61+084932.22 & -1.3 & 14.9 & 0.4545 & 60.7 & 20.96 & -19.96 & 0.90 & 0.4545 & $<0.04$ \\
\hline SDSSJ090519.72+084914.02 & 0.3 & -3.3 & 0.1499 & 6.1 & 22.30 & -16.36 & 0.66 & 0.1501 & $0.82 \pm 0.10$ \\
\hline SDSSJ091845.10+060202.93 & -12.1 & -23.2 & 0.1849 & 56.7 & 18.70 & -19.43 & 1.19 & 0.1849 & $<0.07$ \\
\hline SDSSJ093252.25+073731.59 & 6.4 & 2.5 & 0.3876 & 25.3 & 20.20 & -20.23 & 0.29 & 0.3876 & $1.10 \pm 0.02$ \\
\hline SDSSJ093537.25+112410.66 & 4.0 & 2.6 & 0.2808 & 14.2 & 20.41 & -19.10 & 0.34 & 0.2811 & $0.79 \pm 0.04$ \\
\hline SDSSJ100810.61+014446.17 & 46.5 & -2.8 & 0.2173 & 114.8 & 17.73 & -21.07 & 0.80 & 0.2173 & $<0.20$ \\
\hline SDSSJ100906.91+023557.81 & 8.2 & 2.5 & 0.2523 & 23.7 & 19.21 & -19.85 & 1.13 & 0.2521 & $0.10 \pm 0.01$ \\
\hline SDSSJ102220.71+013143.50 & 25.9 & -35.3 & 0.1369 & 74.3 & 17.45 & -19.99 & 0.99 & 0.1369 & $<0.11$ \\
\hline SDSSJ103605.26+015654.88 & -33.7 & -4.3 & 0.3571 & 119.2 & 18.89 & -21.29 & 0.59 & 0.3571 & $<0.02$ \\
\hline SDSSJ103836.38+095143.68 & -1.8 & 4.8 & 0.1742 & 10.7 & 20.39 & -18.03 & 0.40 & 0.1744 & $1.04 \pm 0.06$ \\
\hline SDSSJ112016.63+093317.94 & -0.4 & -5.6 & 0.4933 & 23.8 & 20.59 & -20.63 & 0.90 & 0.4933 & $2.14 \pm 0.03$ \\
\hline SDSSJ113756.76+085022.38 & -3.9 & 5.2 & 0.3356 & 21.7 & 20.75 & -19.25 & 1.11 & 0.3360 & $0.91 \pm 0.06$ \\
\hline SDSSJ114144.83+080554.09 & 3.1 & -20.7 & 0.2290 & 53.7 & 19.37 & -19.60 & 0.80 & 0.2286 & $0.31 \pm 0.03$ \\
\hline SDSSJ114145.14+080605.27 & 7.7 & -9.5 & 0.3583 & 43.1 & 20.09 & -20.08 & 1.14 & 0.3585 & $0.49 \pm 0.02$ \\
\hline SDSSJ120931.61+004546.23 & -9.7 & -9.7 & 0.2533 & 38.0 & 20.50 & -18.76 & 0.94 & 0.2533 & $<0.06$ \\
\hline SDSSJ122115.84-020259.37 & 7.5 & -6.0 & 0.0934 & 11.7 & 20.65 & -16.22 & 0.51 & & $\ldots$ \\
\hline SDSSJ125737.93+144802.20 & -4.2 & -4.1 & 0.4648 & 24.0 & 20.32 & -20.67 & 1.06 & 0.4644 & $0.12 \pm 0.02$ \\
\hline SDSSJ130555.49+014928.62 & 19.8 & -1.2 & 0.2258 & 50.3 & 19.29 & -19.58 & 0.94 & 0.2258 & $<0.04$ \\
\hline SDSSJ130557.05+014922.34 & 43.2 & -7.5 & 0.1747 & 90.9 & 17.70 & -20.30 & 1.16 & 0.1740 & $0.45 \pm 0.03$ \\
\hline SDSSJ132757.22+101136.02 & -2.8 & -5.8 & 0.2557 & 17.8 & 20.60 & -18.61 & 0.45 & 0.2553 & $0.65 \pm 0.04$ \\
\hline SDSSJ132831.54+075943.00 & 6.8 & 1.0 & 0.3323 & 23.1 & 19.50 & -20.46 & 1.07 & 0.3326 & $0.59 \pm 0.04$ \\
\hline SDSSJ132832.74+075952.56 & 24.7 & 10.6 & 0.2358 & 70.3 & 19.34 & -19.75 & 0.57 & 0.2362 & $0.21 \pm 0.05$ \\
\hline SDSSJ133905.86+002225.36 & 22.8 & 3.4 & 0.1438 & 40.8 & 17.55 & -19.92 & 1.17 & 0.1438 & $<0.22$ \\
\hline SDSSJ140618.34+130143.61 & -18.6 & 36.8 & 0.1748 & 85.6 & 18.08 & -20.04 & 1.00 & 0.1748 & $<0.11$ \\
\hline SDSSJ140619.94+130105.23 & 4.8 & -1.6 & 0.2220 & 12.7 & 19.76 & -19.06 & 0.51 & 0.2222 & $0.96 \pm 0.06$ \\
\hline SDSSJ142600.05-001818.12 & 54.7 & 0.7 & 0.1382 & 93.6 & 15.96 & -21.43 & 1.13 & 0.1382 & $<0.19$ \\
\hline SDSSJ143216.97+095522.23 & 2.8 & 2.9 & 0.3293 & 13.5 & 20.59 & -19.31 & 0.97 & 0.3296 & $2.36 \pm 0.04$ \\
\hline SDSSJ150339.62+064235.04 & -5.4 & -24.9 & 0.2333 & 66.3 & 20.35 & -18.73 & 0.50 & 0.2333 & $<0.06$ \\
\hline SDSSJ150340.15+064308.11 & 2.5 & 8.2 & 0.1809 & 18.2 & 20.57 & -17.47 & 0.97 & 0.1809 & $<0.11$ \\
\hline SDSSJ151228.25-011216.09 & -8.5 & 7.0 & 0.1284 & 17.8 & 19.08 & -18.51 & 0.59 & 0.1284 & $0.94 \pm 0.16$ \\
\hline SDSSJ153112.77+091119.72 & -3.1 & -19.1 & 0.3265 & 63.8 & 20.89 & -19.03 & 0.70 & 0.3265 & $<0.04$ \\
\hline SDSSJ153113.01+091127.02 & 0.4 & -11.8 & 0.2659 & 33.7 & 20.67 & -18.60 & 1.08 & 0.2660 & $0.31 \pm 0.03$ \\
\hline SDSSJ153715.67+023056.39 & 4.9 & 6.7 & 0.2151 & 20.3 & 19.51 & -19.41 & 0.56 & 0.2151 & $0.80 \pm 0.02$ \\
\hline SDSSJ155336.77+053438.23 & 4.6 & 14.3 & 0.3227 & 49.0 & 19.22 & -20.66 & 0.56 & 0.3240 & $0.71 \pm 0.01$ \\
\hline SDSSJ155556.54-003615.58 & -7.9 & -7.2 & 0.3006 & 33.5 & 20.91 & -18.85 & 1.01 & 0.3006 & $<0.04$ \\
\hline SDSSJ160749.54-002228.42 & 3.0 & -8.6 & 0.3985 & 34.1 & 19.63 & -20.88 & 0.84 & 0.3993 & $0.80 \pm 0.01$ \\
\hline SDSSJ160906.36+071330.66 & 14.0 & -6.6 & 0.2075 & 36.8 & 18.93 & -19.63 & 1.10 & 0.2065 & $<0.08$ \\
\hline SDSSJ $204303.53-010139.05$ & -0.3 & -13.0 & 0.2356 & 34.1 & 18.73 & -20.38 & 0.55 & 0.2350 & $1.24 \pm 0.05$ \\
\hline SDSSJ204304.34-010137.91 & 11.8 & -11.9 & 0.1329 & 39.9 & 19.56 & -18.03 & 0.60 & 0.1329 & $<0.19$ \\
\hline SDSSJ210230.86+094121.06 & 2.1 & -4.0 & 0.3565 & 15.8 & 21.05 & -19.12 & 1.53 & 0.3563 & $0.71 \pm 0.04$ \\
\hline SDSSJ212938.98-063758.80 & 5.8 & 3.0 & 0.2782 & 19.4 & 20.94 & -18.49 & 0.92 & 0.2779 & $0.58 \pm 0.03$ \\
\hline SDSSJ $221126.42+124459.93$ & -5.0 & 1.8 & 0.4872 & 22.2 & 20.46 & -20.51 & 0.55 & 0.4840 & $0.40 \pm 0.02$ \\
\hline SDSSJ $221526.04+011353.78$ & -10.5 & -2.7 & 0.3203 & 35.4 & 20.26 & -19.61 & 1.05 & 0.3201 & $0.40 \pm 0.05$ \\
\hline SDSSJ221526.88+011347.20 & 2.1 & -9.3 & 0.1952 & 21.5 & 21.25 & -17.48 & 0.51 & 0.1952 & $<0.15$ \\
\hline SDSSJ222849.01-005640.04 & -2.8 & -9.2 & 0.2410 & 25.5 & 19.80 & -19.27 & 0.88 & & \\
\hline SDSSJ $223246.44+134655.34$ & -5.2 & -6.7 & 0.3221 & 27.9 & 19.19 & -20.68 & 1.00 & 0.3225 & $0.92 \pm 0.05$ \\
\hline SDSSJ223316.34+133315.37 & -7.7 & 5.5 & 0.2138 & 23.1 & 18.85 & -20.08 & 0.69 & 0.2139 & $1.36 \pm 0.06$ \\
\hline SDSSJ223359.74-003320.83 & -2.8 & -5.0 & 0.1162 & 8.5 & 19.86 & -17.37 & 0.78 & 0.1162 & $1.11 \pm 0.09$ \\
\hline SDSSJ224704.01-081601.00 & -11.4 & 16.5 & 0.4270 & 78.7 & 19.84 & -20.88 & 1.34 & 0.4270 & $<0.04$ \\
\hline SDSSJ $230225.06-082156.65$ & -6.4 & -2.5 & 0.3618 & 24.2 & 20.27 & -19.92 & 0.97 & 0.3620 & $2.02 \pm 0.06$ \\
\hline SDSSJ230225.17-082159.07 & -4.7 & -4.9 & 0.2146 & 16.8 & 21.59 & -17.49 & 0.53 & & \\
\hline SDSSJ230845.53-091445.97 & -1.0 & 3.5 & 0.2147 & 8.9 & 19.89 & -18.85 & 0.96 & 0.2139 & $0.43 \pm 0.07$ \\
\hline SDSSJ232812.79-090603.73 & -1.8 & -41.2 & 0.1148 & 60.1 & 18.64 & -18.73 & 0.50 & 0.1148 & $<0.24$ \\
\hline
\end{tabular}

